\title{
Nanobodies provide insight into the molecular mechanisms of the complement cascade and offer new therapeutic strategies
}

\author{
Alessandra Zarantonello ${ }^{1}$, Henrik Pedersen ${ }^{1}$, Nick S Laursen ${ }^{2}$ and Gregers R Andersen ${ }^{1, *}$ \\ 1 Department of Molecular Biology and Genetics, Aarhus University, 8000, Denmark; \\ 2 Department of Biomedicine, Aarhus University, 8000, Denmark; \\ * Correspondence: gra@mbg.au.dk; Tel.: +45 30256646
}

\begin{abstract}
The complement system is part of the innate immune response, where it provides immediate protection from infectious agents and plays a fundamental role in homeostasis. Complement dysregulation occurs in several diseases, where the tightly regulated proteolytic cascade turns offensive. Prominent examples are atypical hemolytic uremic syndrome, paroxysmal nocturnal hemoglobinuria and Alzheimer's disease. Therapeutic intervention targeting complement activation may allow treatment of such debilitating diseases. In this review, we describe a panel of complement targeting nanobodies that allow modulation at different steps of the proteolytic cascade, from the activation of the $\mathrm{C} 1$ complex in the classical pathway to formation of the $\mathrm{C} 5$ convertase in the terminal pathway. Thorough structural and functional characterization has provided a deep mechanistic understanding of the mode of inhibition for each of the nanobodies. These complement specific nanobodies are novel powerful probes for basic research and offer new opportunities for in vivo complement modulation.
\end{abstract}

Keywords: complement system; proteolytic cascade; convertase; inhibitor; structural biology; molecular mechanism

Introduction

1.1 The complement system

In the following, we describe those pathways and molecules from the complement system that are needed to understand the experimental results described in the upcoming sections. For more comprehensive reviews of specific aspects, the reader is referred to recent review works including [2-4].The complement system is an efficient weapon of innate immunity, which opsonizes the surface of invading organisms and apoptotic host cells for elimination through phagocytosis and cell lysis. In the innate immune system, pattern recognition molecules bind pathogen-associated molecular patterns and damageassociated molecular patterns $[4,6]$. Complement activation also elicits an inflammatory response at the site of infection. The complement cascade can be activated through three distinct pathways; the classical pathway (CP), the lectin pathway (LP), and the alternative pathway (AP) (Figure 1). Both the classical and the lectin pathways initiate by activation of giant complexes formed between an oligomeric PRM and a protease complex that cleaves complement component $\mathrm{C} 4$ resulting in deposition of the major fragment $\mathrm{C} 4 \mathrm{~b}$ on the activator (Figure 1). In the $\mathrm{CP}, \mathrm{C} 1 \mathrm{q}$ is the pattern recognition molecule and circulates in complex with the serine proteases $\mathrm{C} 1 \mathrm{r}$ and $\mathrm{C} 1 \mathrm{~s}$ ( $\mathrm{C} 1$ in Figure 1). $\mathrm{C} 1 \mathrm{q}$ is a hexamer of trimers, and each trimer is formed by chains A, B and C. The C-terminal parts of the three chains fold into structural entities known as the $\mathrm{C} 1 \mathrm{q}$ globular domains, while the $\mathrm{N}$ - 
terminal parts of the three chain are joined in a collagen helix. The $\mathrm{C} 1$ complex initiates the $\mathrm{CP}$ when $\mathrm{C} 1 \mathrm{q}$ recognizes antigen bound $\mathrm{IgG} / \mathrm{IgM}$ (Figure 1), the acute phase proteins $\mathrm{CRP}$, pentraxins and anionic phospholipids as phosphatidylserine on apoptotic and necrotic cell surfaces $[7,8]$. C1q similarly recognizes LPS in the cell wall of Gram-negative bacteria as well as viral proteins [9]. In the brain, $\mathrm{C} 1 \mathrm{q}$ binds $\mathrm{A} \beta$ and PrP oligomers, playing a role in Alzheimer's and prion disease progression, respectively [10, 11]. The vast majority of $\mathrm{C} 1 \mathrm{q}$ binding patterns are recognized by the $\mathrm{C} 1 \mathrm{q}$ globular domain. Specifically, the $\mathrm{C} 1 \mathrm{q} B$ and $\mathrm{C}$ chains establish direct interactions with the $\mathrm{Fc}$ of IgG and IgM in immune complexes [1, 4, 7, 12-15]. Activated $\mathrm{C} 1 \mathrm{~s}$ in the $\mathrm{C} 1$ complex cleaves $\mathrm{C} 4$ to $\mathrm{C} 4 \mathrm{~b}$, and a major conformational change in nascent $\mathrm{C} 4 \mathrm{~b}$ exposes a reactive thioester group, which may react with a nucleophile leading to covalent attachment of $C 4 b$ to the activator (Figure 1) [16, 17]. The $\mathrm{C} 4 \mathrm{~b}$ conformation allows binding of the zymogen $\mathrm{C} 2$, to form the proconvertase $\mathrm{C} 4 \mathrm{~b} 2$. Within the proconvertase, $\mathrm{C} 2$ is cleaved by $\mathrm{C} 1 \mathrm{~s}$ resulting in formation of the $\mathrm{C} 3$ convertase $\mathrm{C} 4 \mathrm{~b} 2 \mathrm{a}$ [18-20]. In the related LP, the cascade can be initiated by five different PRMs called mannan-binding lectin (MBL), M-, L-, H-ficolins (or ficolin-1 to 3) and CLLK [21-23] upon binding to conserved carbohydrate structures on the activator surface. The LP PRMs circulate in complex with dimers of the MBL-associated serine proteases (MASPs) 1, 2, and 3 [24-26]. MASP-1 and MASP-2 are functional homologs of C1r and C1s in the $\mathrm{CP}$, and upon activation of the LP cascade, C4 is cleaved and the same proconvertase and C3 convertase are assembled as in the CP. Since the C4b2a C3 convertase is the endpoint of both pathways, it is referred to as the $\mathrm{CP} / \mathrm{LP} \mathrm{C} 3$ convertase. C4b2a cleaves its substrate $\mathrm{C} 3$, which undergoes a conformational change similar to nascent $\mathrm{C} 4 \mathrm{~b}$, and exposes the reactive thioester that forms an ester bond with a hydroxyl nucleophile on the surface (Figure 1) [27, 28].

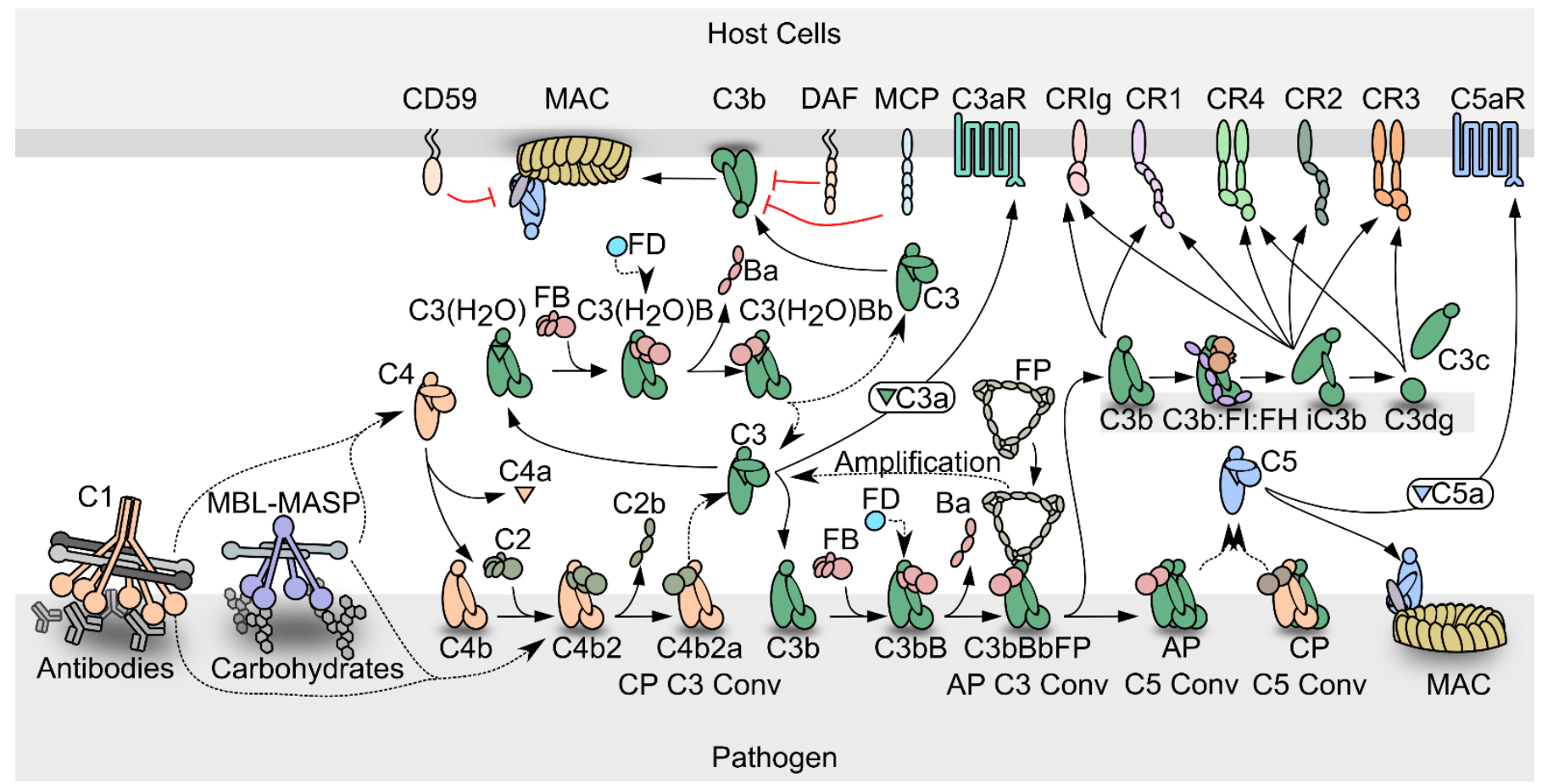

Figure 1 Schematic representation of the complement system. PRM-associated proteases activate upon binding of the PRMs to specific activators. The active proteases cleave $\mathrm{C} 4$, which undergoes a conformational change and covalently binds the activator. The zymogen $\mathrm{C} 2$ binds the opsonizing $\mathrm{C} 4 \mathrm{~b}$, forming the $\mathrm{C} 3$ proconvertase. The active $\mathrm{C} 3$ convertase cleaves $\mathrm{C} 3$, which liberates the $\mathrm{C} 3 \mathrm{a}$ 
fragment from $\mathrm{C} 3 \mathrm{~b}$ that covalently attaches to the activator. FB may bind the $\mathrm{C} 3 \mathrm{~b}$, and upon activation by Factor D, the AP C3 convertase appears, that is stabilized by properdin (FP). C3 may also undergo spontaneous hydrolysis forming C3(H2O), which is a functional homologue of $\mathrm{C} 3 \mathrm{~b}$ and allows the assembly of a fluid phase $\mathrm{C} 3$ convertase. When the C3b concentration reaches a threshold density on the activator, the $\mathrm{C} 3$ convertases shifts specificity to $\mathrm{C} 5$. The resulting $\mathrm{C} 5$ convertases cleave $\mathrm{C} 5$ and the $\mathrm{C} 5 \mathrm{~b}$ forms the starting point for assembly of the membrane attack complex that may perforate the cell membrane. C3b may also undergo degradation by factor I assisted by cofactors. The degradation of C3b opens for interactions with complement receptors.

In the alternative pathway, $\mathrm{C} 3 \mathrm{~b}$ deposited by the $\mathrm{CP} / \mathrm{LP} \mathrm{C} 3$ convertase binds to factor $\mathrm{B}(\mathrm{FB})$ to form the AP proconvertase $\mathrm{C} 3 \mathrm{bB}$ (Figure 1). The fluid phase protease Factor D (FD) cleaves FB in the proconvertase and the AP C3 convertase C3bBb appears [29-31]. The ability of $\mathrm{C} 3 \mathrm{~b}$ to form a $\mathrm{C} 3$ convertase and promote further $\mathrm{C} 3$ cleavage gives rise to the AP amplification loop, which amplifies the C3b deposition catalyzed by the CP/LP C3 convertase by 5-10 fold [32-34]. Besides starting from C3b deposited through the $\mathrm{CP}$ and LP, a fluid phase AP C3 convertase may assemble after spontaneous C3 hydrolysis (tickover mechanism) whereby a C3b-like molecule called $\mathrm{C} 3\left(\mathrm{H}_{2} \mathrm{O}\right)$ is formed that is capable of binding to FB [35]. This fluid phase proconvertase is also activated by FD cleavage, however the physiological role of the fluid phase AP C3 convertase is still debated [36, 37]. The half-life of the AP C3 convertase is only $90 \mathrm{~s}$ under physiological conditions, but it is increased up to ten-fold by binding of properdin (FP), the only known positive regulator of the complement system [38, 39]. FP circulates as dimeric, trimeric, or tetrameric homo-oligomers and recognizes primarily $\mathrm{C} 3 \mathrm{~b}$ within the AP C3 convertase [40].

Both $\mathrm{C} 4 \mathrm{~b}$ and $\mathrm{C} 3 \mathrm{~b}$ are tightly regulated to avoid complement activation on host cells, but due to the strong amplification through the alternative pathway, $\mathrm{C} 3 \mathrm{~b}$ regulation is likely to be the most important in vivo. Regulators of complement activity (RCA) expressed on or recruited to host cells may exert decay acceleration by dissociating the C3 convertases, but may also act as cofactors for $\mathrm{C} 4 \mathrm{~b}$ and $\mathrm{C} 3 \mathrm{~b}$ degradation by the protease factor I (FI) [41] (Figure 1). The products of FI degradation, C4c C4d, iC3b and C3dg are inactive with respect to convertase formation. The best characterized FI cofactors are factor $\mathrm{H}(\mathrm{FH})$, membrane cofactor protein (MCP/CD46) and CR1 (CD35). MCP and CR1 are membrane bound and recognize both $\mathrm{C} 4 \mathrm{~b}$ and $\mathrm{C} 3 \mathrm{~b}[42,43]$. In contrast, $\mathrm{FH}$ is $\mathrm{C} 3 \mathrm{~b}$ specific and acts both in the fluid phase and on host cells through recognition of sialic acid and glycosaminoglycan [44, 45]. On non-host cell like pathogens, conversion of C3b to $\mathrm{iC} 3 \mathrm{~b}$ is slower than on host cells due to weaker binding of FH and the lack of membrane bound regulators.

The multiple effector functions elicited by the $\mathrm{C} 3$ degradation products are reviewed in [46]. C3 cleavage releases the anaphylatoxin C3a, which triggers an inflammatory response at the activation site through binding to the G-protein coupled receptor C3aR [47]. Another effector function of $\mathrm{C} 3$ fragments is to induce phagocytosis of the activator. $\mathrm{C} 3 \mathrm{~b}$ and $\mathrm{iC} 3 \mathrm{~b}$ mediate phagocytosis through interaction with complement receptor Vsig4 (also called CRIg) presented by Kupffer cell macrophages [48, 49], while only iC3b are recognized by CR3 and CR4 to elicit phagocytosis. CR3 and CR4 are expressed in the myeloid subsets of leukocytes, on NK cells and activated T and B lymphocytes [50]. Furthermore, $\mathrm{iC} 3 \mathrm{~b}$ and its degradation product $\mathrm{C} 3 \mathrm{dg}$ confer cross talk between the 
complement cascade and adaptive immunity through their binding to CR2 on B lymphocytes [51].

If not degraded by $\mathrm{FI}$, the surface density of $\mathrm{C} 3 \mathrm{~b}$ continues to increase on the activator. When a threshold density is reached, both $\mathrm{C} 3$ convertases shift their substrate specificity from $C 3$ to $C 5[52,53]$. C5 is structurally homologous to complement $C 3$ [54], but does not have an internal thioester. Instead, C5b interacts with C6, forming the C5b6 complex, which can transiently associate with a nearby lipid bilayer on a complement opsonized cell. Subsequent recruitment of $\mathrm{C} 7$ leads to stable association with the membrane, and lipid bilayer penetration starts when C8 joins the complex. The pore size is increased by insertion of several C9 molecules, resulting in formation of the membrane attack complex (MAC) that perforates the cell (Figure 1) [55]. The lytic ability of complement is important for killing of Gram negative bacteria, one important example is Neisseria meningitidis [56]. The cleavage of $\mathrm{C} 5$ also leads to the release of $\mathrm{C} 5 \mathrm{a}$, which triggers a potent inflammatory response through binding to the G-protein coupled receptor C5aR1. This induces vasodilation, release of histamine and contraction of smooth muscle, as well as chemotaxis of neutrophils, T cells, activated B cells, macrophages and basophils [57].

\subsection{The complement system as a driver of pathogenesis}

Regulators of complement are ubiquitously expressed on host surfaces to control undesired amplification, and tipping of this balance is a major mechanism for diseases associated with complement activation. It is well established that especially aberrant AP activity on self surfaces is associated with the development of atypical hemolytic uremic syndrome (aHUS), paroxysmal nocturnal hemoglobinuria (PNH), age related macular degeneration (AMD), anti-neutrophilic cytoplasmic autoantibodies (ANCA) vasculitis, and C3 glomerulopathy (C3G) [46]. PNH arises from clonal expansion of hematopoietic stem cells that comprise a loss-of-function mutation in the PIGA gene [58] that is essential for synthesis of GPI anchors and the mutation hence results in deficiency of GPI anchored proteins, including DAF [59] and CD59 [60]. Lack of these surface bound complement regulators render erythrocytes vulnerable to complement attack and lysis by the membrane attack complex. The clinical manifestations of the disease include thrombosis and anemia [58]. Similarly, complement exacerbates the development of aHUS that arises from dysregulation of complement on host endothelia, most commonly the kidneys [61]. Patients suffering from aHUS often possess mutations in FH [62] which may result in reduced levels of surface-bound $\mathrm{FH}$ and consequently reduced protection against complement activation. Similarly, genetic studies report loss-of-function mutations in genes encoding the complement regulators FI and $\mathrm{MCP}$ as well as gain-of-function mutations in genes for FB and C3 in aHUS patients [63]. The term ' $\mathrm{C} 3$ glomerulopathy' is used to describe glomerular disorders, where complement dysregulation either underlies or exacerbates disease development [64]. A common characteristic of C3 glomerulopathies $(\mathrm{C} 3 \mathrm{G})$ is $\mathrm{C} 3$ fragment deposition in the renal tissue leading to irreversible kidney damage. C3G arise from dysregulation of the AP in fluid phase driven by either acquired or genetic factors. Acquired drivers include autoantibodies, called C3 nephritic factors (C3Nef), that stabilize the C3 convertase [65]. In the dense deposit disease subtype of C3G, 78\% of patients express C3Nefs [66]. Similarly, genetic drivers of C3G commonly lie in the C3 and 
CFB as well as CFH loci [67]. AMD is the leading cause of visual impairment in developed countries. In the early stage of disease development, extracellular deposits of lipids and proteins accumulate between the retinal pigment epithelium and the Bruch's membrane. Later stages of the disease result in extensive damage of the retinal pigment epithelium and eventually loss of vision [68]. An analysis of genetic data from more than 17,000 cases indicated an association between disease and mutations near the genes encoding FH, FI, FB, C2 and C3 [69].

The contexts in which the $\mathrm{CP}$ of complement is involved are broad, from the homeostatic removal of apoptotic material to induction of heavy inflammation in host tissues. On host cells, tagging by the $\mathrm{C} 1$ complex leads to signaling for clearance of the debris. Importantly, this takes place in the absence of inflammation and lysis, due to the action of FI and the regulators, which promptly degrade $\mathrm{C} 3 \mathrm{~b}$ to $\mathrm{iC} 3 \mathrm{~b}$ and dissociates the convertases [70]. It is well-established that deficiency in the early $\mathrm{CP}$ components leads to autoimmunity and development of autoantibodies against neoepitopes on the surface of apoptotic cells, due to impaired clearance of apoptotic material [71]. Furthermore, autoimmunity caused by $\mathrm{CP}$ activation is also observed in the acute diseases ischemia reperfusion injury, sepsis, antibody induced hemolytic anemia, antibody mediated rejection and cold agglutinin disease [72-76].

In recent years, it has been established that the classical pathway has a well-defined role in the developmental process of synaptic pruning [77], a process required in the developing brain to establish proper synaptic connectivity for a functioning adult brain. Less active synapses are pruned away and one of the mechanisms guiding this process is activation of the complement system through the classical pathway with C4 cleavage, progressing further into the alternative pathway resulting in C3 cleavage [78, 79]. In the neurodevelopmental disorder schizophrenia, genome wide association studies have shown a correlation of a C4 isotype overexpression with disease development, and a polymorphism in the CNS specific functional homologue of CR1, CSMD1 was identified as a risk factor [80-82]. Most recently, $\mathrm{C} 4$ overexpression was linked to hypo-connectivity in the prefrontal cortex, and schizophrenia-like symptoms in mice [83], and the current hypothesis for schizophrenia pathogenesis involves aberrant complement mediated synaptic pruning. Synaptic pruning onset was also documented during West Nile virus infection and in the neurodegenerative diseases Alzheimer's, Parkinson's, multiple sclerosis, frontotemporal dementia and spinal muscular atrophy [84-87]. Hence, the normal function of the $\mathrm{CP}$ during development can be aberrantly reactivated with devastating consequences in adulthood $[79,84-86,88]$ and for this reason the $\mathrm{CP}$ proteins represent promising therapeutic targets for treatment of neurological diseases with different etiology [87].

\section{The complement targeting nanobodies}

\subsection{Complement specific nanobodies for therapy, research and diagnostics}

The examples presented above underscore the complement system as a therapeutic target, and for more than a decade, there has been significant efforts with respect to de- 
veloping therapeutic molecules for control of the complement cascade based on e.g. antibodies, pathogen complement evasion proteins, small molecules, peptides, and gene therapy $[89,90]$. In addition, new reagents for capture and detection of complement proteins may improve diagnostics for the evaluation of future complement therapeutics, which currently relies on the assessment of complement levels, functional assessment of specific pathways and detection of complement degradation products [91]. The involvement of complement in diseases such as Alzheimer's disease and schizophrenia remains enigmatic. Administration of pathway-specific inhibitors to the brain parenchyma in murine models may allow an accurate mapping of reactions and interactions in the complement system underlying neurological diseases, but delivery across the blood-brain barrier of antibodies and other complement therapeutics represents a severe challenge.

Single domain antibodies derived from heavy chain only antibodies found in animals such as llamas (llama glama) called nanobodies [92], are attractive but also largely unexplored modalities for functional analysis and therapeutic control of the complement system. Protocols for selection and development of nanobodies are well established [93]. In the past years, we have developed a portfolio of high-affinity nanobodies specific for proteins in the complement system. A lama is immunized with four injections of $500 \mu \mathrm{g}$ of the complement antigen, in intervals of three weeks. We isolate the peripheral blood lymphocytes from the immunized lama, extract the RNA, and generate cDNA. This allows us to construct phage display libraries and select nanobodies using in vitro panning. In the following, we provide an overview of complement targeting nanobodies developed by us and others.

\subsection{The complement deposition assays and hemolytic assays}

We and others have evaluated the activity of complement inhibitors, including nanobodies, using multiple complement deposition assays (Figure 2A). The typical assay starts by coating the surface with the complement activator of choice, which depends on the pathway investigated. For the $\mathrm{CP}$, the wells are coated with heat aggregated normal human IgG [94], for the LP they are coated with mannan [95, 96], a cell wall polysaccharide found in yeast. In assays for AP activity, the wells are coated with zymosan [97], a glucan formed by beta-1,3-glycosidic linkages, simulating the coating of a bacterial surface. The source of complement is normal human serum, which is added in each well at a given dilution depending on the dynamic range of the assay, together with a titration of the nanobody under investigation. Activation leading to deposition is carried out at $37^{\circ} \mathrm{C}$ for $90 \mathrm{~min}$, after which the plate is washed and the deposition of complement fragments is detected by addition of a polyclonal antibody specific for $\mathrm{C} 3 \mathrm{c}$ or $\mathrm{C} 4 \mathrm{c}$, conjugated to biotin. 
The readout of the assay are the fluorescence counts generated by europium-conjugated streptavidin. If the counts number is comparable to the counts obtained in the absence of the nanobody, the nanobody is non-inhibitory, while if they are lower, the nanobody has inhibitory activity (Figure 2A, right). Another useful assay for evaluation of complement activation monitors hemolysis of sheep red blood cells sensitized with IgG or IgM antibodies. The red blood cells are lysed only if the MAC complex is assembled on their surface, which is only possible if the CP C5 convertase or the AP C5 convertase formed through the AP amplification loop cleave C5 (Figure 2B). Absence of hemolysis confirms that the nanobody inhibits assembly of the MAC directly or indirectly by inhibiting one of the upstream pathways.

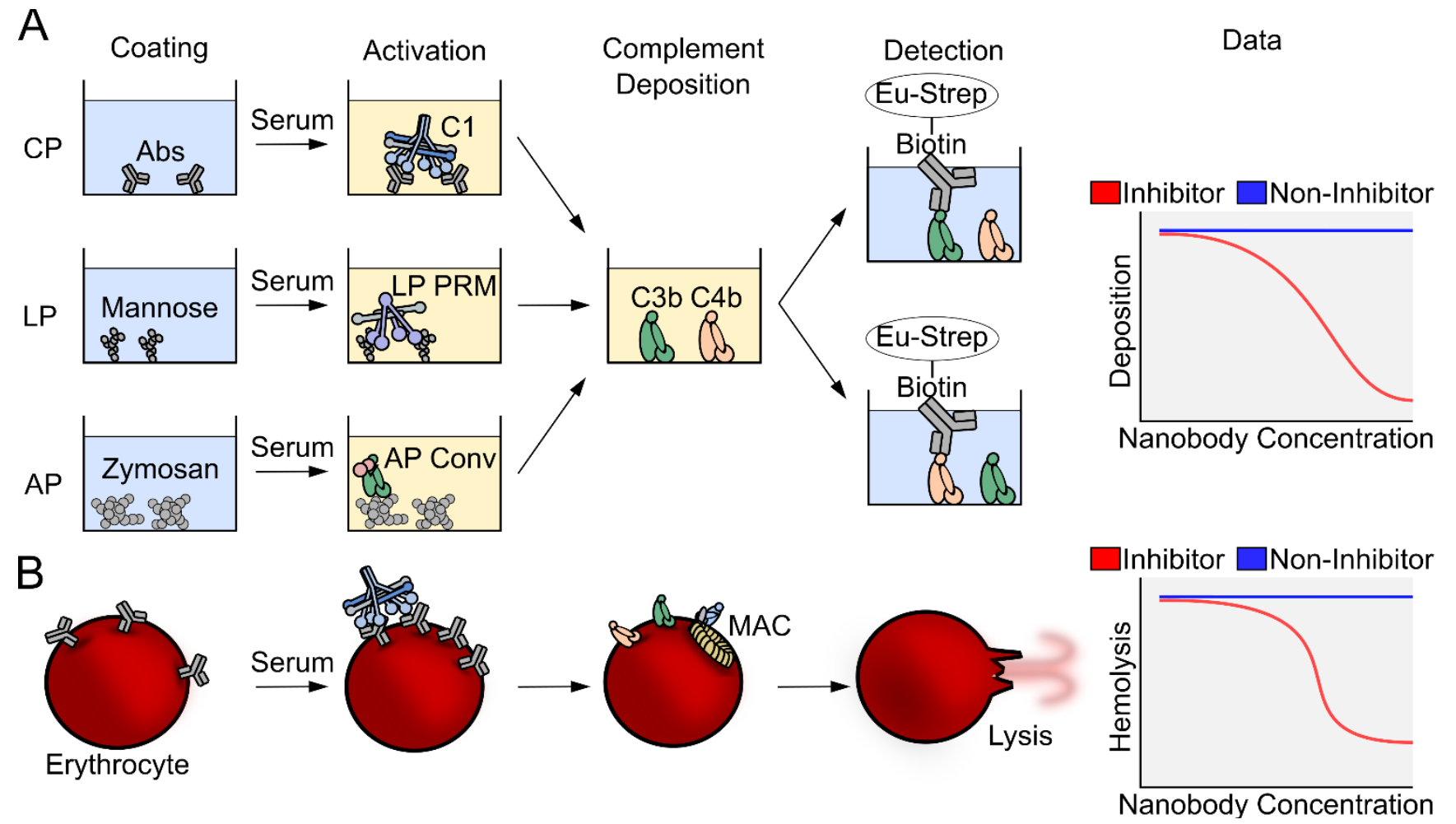

Figure 2. Overview of complement assays. A) In ELISA based complement deposition assays, pathway-specific molecular patterns activate either the $\mathrm{CP}$, the LP or the AP. The covalent binding of complement components allows thorough washing and subsequent readout of either C4 or C3 deposition. B) CP activation can be provoked on sheep erythrocytes using specific antibodies. The complement system may progress to the terminal pathway and the assembly of the membrane attack complex that lyses the erythrocytes. The amount of hemoglobin released can be measured by spectrophotometry at $405 \mathrm{~nm}$ for quantification of the fraction of lysed cells.

\subsection{C1qNb75 inhibits initiation of the classical pathway}

The C1q specific nanobody C1qNb75 was selected from a phage display library generated after immunization of a lama with human C1q [5]. C1qNb75 was chosen among a panel of C1q specific nanobodies based on its ability to inhibit CP activation by IgG when analyzed in C4 and C3 fragment deposition assays (Figure 3A). C1qNb75 binds with high 
affinity (dissociation constant, $\mathrm{K}_{\mathrm{D}}=0.3 \mathrm{nM}$, Table 1) to the isolated recombinant globular domain of $\mathrm{C} 1 \mathrm{q}$ suggesting that it may prevent CP activation by occluding the IgG binding site on C1q. Indeed, the nanobody directly blocks C1q binding to IgG coated surfaces and to IgG-antigen immune complexes in a concentration dependent manner. Interestingly, in the $\mathrm{C} 3$ deposition assay a 6-fold molar ratio of $\mathrm{C} 1 \mathrm{qNb} 75$ to $\mathrm{C} 1 \mathrm{q}$ was necessary to fully inhibit CP activation, indicating that all IgG Fc binding sites on the hexameric C1q need to be occupied by the nanobody. We also validated the CP inhibitory activity of the $\mathrm{C} 1 \mathrm{qNb} 75$ nanobody with respect to the propagation to the terminal pathway in hemolysis assays. Sheep red blood cells were either coated with IgG or IgM, and release of hemoglobin was measured in the presence of $\mathrm{C} 1 \mathrm{qNb} 75$. In accordance with the inhibition of $\mathrm{C} 3$ deposition, C1qNb75 also prevented hemolysis (Figure 3B). Finally, to obtain the structural basis for $\mathrm{C} 1 \mathrm{qNb} 75$ inhibition of the $\mathrm{CP}$, we determined the structure of the nanobody in complex with the C1q globular domain [5]. The resulting crystal structure determined at $2.2 \AA$ resolution demonstrated that $\mathrm{C} 1 \mathrm{qNb} 75$ binds to chain $\mathrm{B}$ and chain $\mathrm{C}$ of $\mathrm{C} 1 \mathrm{q}$ and revealed that $\mathrm{C} 1 \mathrm{qNb} 75$ acts by imposing steric hindrance on the $\mathrm{C} 1 \mathrm{q}$ globular domain such that the interaction with the Fc of $\operatorname{IgG}$ and $\operatorname{IgM}$ is prevented (Figure 3C-E).

Table 1. The complement targeting nanobodies presented in this review. The table outlines the inhibitory mechanism of each nanobody, its epitope and antigen. Dissociation constants are given toward different forms of the targeted complement component, and toward the mouse component when available.

\begin{tabular}{|c|c|c|c|c|}
\hline $\begin{array}{l}\text { Nanobody (ref- } \\
\text { erence) }\end{array}$ & Mechanism & Epitope & Antigen & $\begin{array}{l}\text { Dissociation con- } \\
\text { stant } K_{D}(\mathrm{nM})\end{array}$ \\
\hline C1qNb75 [5] & $\begin{array}{c}\text { Prevents } \mathrm{C} 1 \mathrm{q} \text { binding to } \mathrm{Fc} \text { of } \operatorname{IgG} \\
\text { and } \operatorname{IgM}\end{array}$ & gC1q chains B and C & hgC1q & 0.3 \\
\hline \multirow[t]{3}{*}{ hC4Nb8 [98] } & \multirow[t]{3}{*}{ Blocks CP proconvertase assembly } & \multirow{3}{*}{$\begin{array}{c}\text { C4b } \alpha^{\prime}-\mathrm{Nt}, \mathrm{MG} 6 \text { and MG7 do- } \\
\text { mains }\end{array}$} & $\mathrm{hC4}$ & 2000 \\
\hline & & & $\mathrm{hC} 4 \mathrm{~b}$ & 0.016 \\
\hline & & & $\mathrm{mC} 4 \mathrm{~b}$ & 0.2 \\
\hline \multirow[t]{2}{*}{ hC3Nb1 [99] } & \multirow[t]{2}{*}{$\begin{array}{l}\text { Blocks AP proconvertase assem- } \\
\text { bly }\end{array}$} & \multirow[t]{2}{*}{$\begin{array}{l}\text { C3 and C3b MG6 and MG7 } \\
\text { domain }\end{array}$} & $\mathrm{hC3}$ & 0.9 \\
\hline & & & $\mathrm{hC} 3 \mathrm{~b}$ & 0.2 \\
\hline \multirow[t]{4}{*}{ hC3Nb2 [100] } & \multirow{4}{*}{$\begin{array}{l}\text { Prevents substrate binding to } \mathrm{C} 3 \\
\text { convertases }\end{array}$} & \multirow{4}{*}{$\begin{array}{c}\text { C3 and C3b MG3 and MG4 } \\
\text { domains }\end{array}$} & $\mathrm{hC} 3$ & 10 \\
\hline & & & $\mathrm{hC} 3 \mathrm{~b}$ & 5 \\
\hline & & & hC3-MA & 3 \\
\hline & & & $\mathrm{mC} 3 \mathrm{~b}$ & 0.6 \\
\hline \multirow[t]{2}{*}{ hC3Nb3 [101] } & \multirow{2}{*}{$\begin{array}{l}\text { Blocks AP C3 proconvertase as- } \\
\text { sembly and CP C5 convertase ac- } \\
\text { tivity }\end{array}$} & \multirow[t]{2}{*}{ C3 and C3b C345c domain } & $\mathrm{hC3}$ & 3 \\
\hline & & & $\mathrm{hC} 3 \mathrm{~b}$ & 3 \\
\hline
\end{tabular}




\begin{tabular}{|c|c|c|c|c|}
\hline & & & hC3-MA & 6 \\
\hline & & & $\mathrm{mC} 3 \mathrm{~b}$ & 3 \\
\hline hFPNb1 [40] & Non-inhibitory & FP thrombospondin repeat 4 & hFPc & 7.3 \\
\hline \multirow[t]{3}{*}{ Nb119 [102] } & Blocks $\mathrm{C} 3 \mathrm{~b}$ and $\mathrm{C} 3 \mathrm{c}$ binding to & Vsig4 (CRIg) ectodomain & hVsig4 & 850 \\
\hline & Vsig4 (CRIg) & & & \\
\hline & & & mVsig4 & 3.5 \\
\hline
\end{tabular}


A

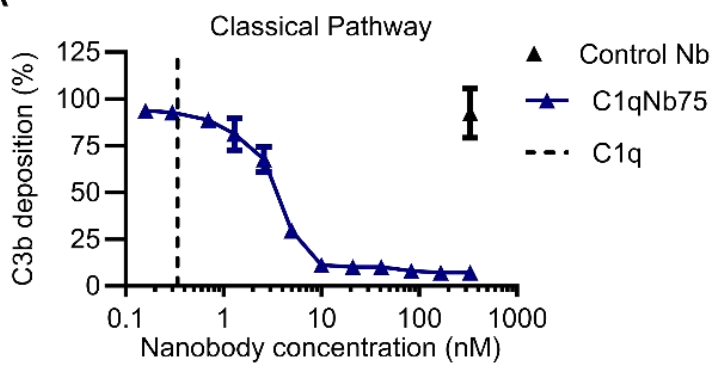

B
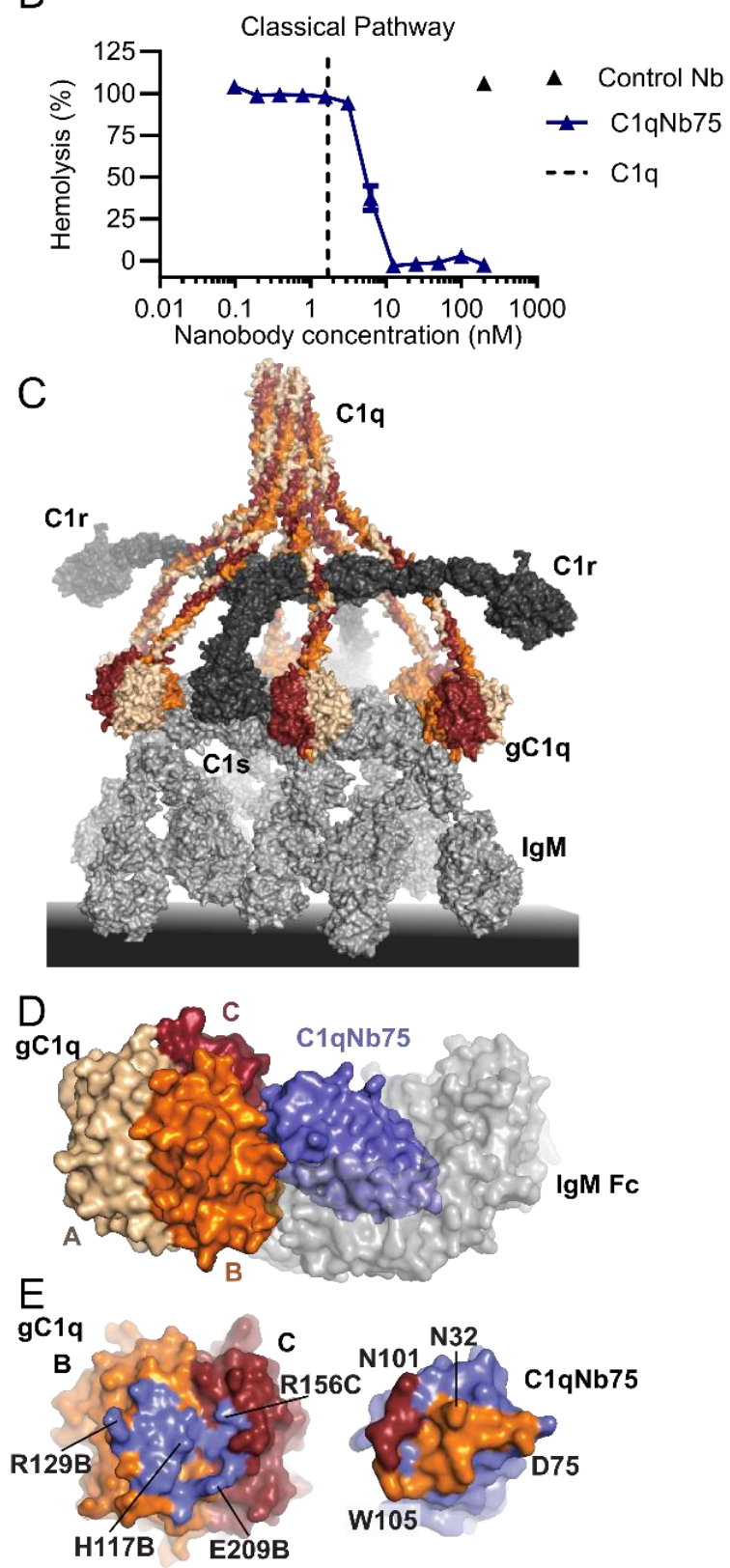

In conclusion, the data show that $\mathrm{C} 1 \mathrm{qNb} 75$ is a potent inhibitor of antibody-mediated $\mathrm{CP}$ activation. $\mathrm{C} 1 \mathrm{qNb} 75$ may thus represent a candidate for further development of therapeutic molecules for treatment of diseases where IgG/IgM autoantibodies mediate excessive complement activation. C1q binds a multitude of ligands besides IgG and IgM, ranging from patterns on synapses during development and disease, to acute phase proteins like CRP and pentraxin 3. We do not currently know which $\mathrm{C} 1 \mathrm{q}$ interactions besides those formed with IgG and IgM the nanobody inhibits. C1qNb75 may however be used as a convenient tool to probe the regions on $\mathrm{C} 1 \mathrm{q}$ involved in recognition of these non-antibody ligands. Unfortunately, the nanobody is not cross reactive towards murine $\mathrm{C} 1 \mathrm{q}$ but may react with C1q from non-human primates [5]. Nonetheless, C1qNb75 may still be useful in transgenic murine disease models where the human genes for the three $\mathrm{C} 1 \mathrm{q}$ subunit fully or partially replaces the murine $\mathrm{C} 1 \mathrm{q}$ genes. Such a chimeric $\mathrm{C} 1 \mathrm{q}$ was recently used to evaluate the in vivo potential of a bispecific antibody allowing the targeting of human $\mathrm{C} 1 \mathrm{q}$ to infectious microorganisms and cancer cells [103].

\section{4 hC4Nb8 inhibits in vivo assembly of the CP and LP C3 convertase}

Moving to the next step of the classical pathway, a target which is shared with the LP is the proconvertase $\mathrm{C} 4 \mathrm{bC} 2$. Inhibition at this stage will not affect the $\mathrm{C} 1 \mathrm{q}$ functions independent of $\mathrm{CP}$ downstream activation [104], but will attenuate both the CP and LP cascades before $\mathrm{C} 3$ cleavage takes place. Hence, an inhibitor of the $\mathrm{CP}$ C3 convertase is predicted to tune down the effects of complement activation in diseases linked to CP and LP over-activation, while not affecting basal AP activity (Figure 1). The nanobody hC4Nb8 was selected against human $\mathrm{C} 4 \mathrm{~b}$ by phage display after immunization of a

Figure 3. Structure and function $\mathrm{C} 1 \mathrm{qNb} 75$. A) Effect of $\mathrm{C} 1 \mathrm{qNb} 75$ or control $\mathrm{Nb}$ on $\mathrm{C} 3$ fragment deposition in $0.2 \% \mathrm{NHS}$. The $\mathrm{CP}$ was activated by heat aggregated IgG. B) Hemolysis of IgM coated sheep erythrocytes in the presence of C1qNb75 or control Nb in $1 \%$ NHS. The putative $\mathrm{C} 1 \mathrm{q}$ concentration in the assay is shown as a dashed line. C) Structural model of the C1 complex bound to IgM based on [1]. The three chains of $\mathrm{C} 1 \mathrm{q}$ is shown in wheat, orange and dark red. C1r and C1s in dark grey and IgM in light grey. D) Structure of the globular domain of $\mathrm{C} 1 \mathrm{q}(\mathrm{gC1q})$ in complex with C1qNb75 (blue) [PDB entry 6Z6V]. IgM-Fc ( semi-transparent grey surface) is shown to illustrate the steric clash between $\mathrm{C} 1 \mathrm{qNb} 75$ and IgM. Adapted from [5]. E) Epitope of C1qNb75 (left) on gC1q shown in blue and paratope on C1qNb75 (right). The paratope on $\mathrm{C} 1 \mathrm{qNb} 75$ is colored according to the $\mathrm{C} 1 \mathrm{q}$ chain recognized. 
lama with human $\mathrm{C} 4 \mathrm{~b}$. To screen for the presence of complement inhibitors, $\mathrm{CP}$ deposition assays were performed and evaluated at the level of C4 and C3 fragment deposition in the presence of the nanobody [98]. In these assays, hC4Nb8 inhibited C3 fragment deposition in a dose dependent manner without influencing C4 deposition (Figure 4A-B). After preliminary experiments showing that the nanobody inhibits $\mathrm{C} 4 \mathrm{~b} 2$ formation in a size exclusion chromatography assay, $\mathrm{hC} 4 \mathrm{Nb} 8$ in complex with $\mathrm{C} 4 \mathrm{~b}$ was crystallized and the structure of the complex was determined at $3.3 \AA$ resolution [98]. Both complement C3 and C4 comprise eight macroglobulin-like (MG) domains, a C1r/C1s, Uegf, Bmp1 (CUB) domain, a thioester domain (TE), an anaphylatoxin (ANA) domain and a C-terminal C345c domain (Figure 4C). Proteolytic activation of $\mathrm{C} 3$ and $\mathrm{C} 4$ to $\mathrm{C} 3 \mathrm{~b}$ and $\mathrm{C} 4 \mathrm{~b}$, respectively, releases the ANA domain and leads to a dramatic conformational change $[16,17,27,28]$ that allows the thioester in nascent $\mathrm{C} 4 \mathrm{~b}$ and $\mathrm{C} 3 \mathrm{~b}$ to react with a nucleophile on the activator. The atomic model of $\mathrm{C} 4 \mathrm{~b}$ in complex with $\mathrm{hC} 4 \mathrm{Nb} 8$ revealed that the epitope of the nanobody encompasses residues in an N-terminal region of the $\alpha^{\prime}$-chain of $\mathrm{C} 4 \mathrm{~b}$, along with some residues on the MG6 and MG7 domains (Figure 4D and F). This is a neoepitope generated upon C4 cleavage and is part of the binding site for C2 [105]. In agreement with this, the nanobody binds with a dissociation constant of $16 \mathrm{pM}$ to $\mathrm{C} 4 \mathrm{~b}$, a $10^{5}$-fold higher affinity compared to native C4 ( $2 \mu \mathrm{M}$, Table 1$)$. The steric hindrance imposed by hC4Nb8 on C2 binding was confirmed by comparison of the $\mathrm{C} 4 \mathrm{~b} 2$ proconvertase model obtained from SAXS data [106] (Figure 4E) and a 3D reconstruction of C4b2 obtained by electron microscopy with the crystal structure of the C4b:hC4Nb8 complex [98]. However, hC4Nb8 also inhibited CR1 mediated FI cleavage of C4b, in agreement with the predicted steric overlap of hC4Nb8 with the putative binding site for CR1 on C4b $[45,98,107]$.

The atomic structure of the $\mathrm{C} 4 \mathrm{~b}: \mathrm{hC} 4 \mathrm{Nb} 8$ complex in combination with the functional data provided a thorough mechanistic basis for understanding how complement inhibition is exerted by hC4Nb8. To test whether hC4Nb8 could control CP activation in vivo, we took advantage of a transgenic hC4 knock-in mouse model and performed a passive immunization experiment. The nanobody was injected subcutaneously along with the high molecular weight red fluorescent antigen phycoerythrin, allowing local CP activation, resulting in $\mathrm{C} 4$ and $\mathrm{C} 3$ deposition on immune complexes formed between antibodies and the phycoerythrin antigen. Such iC $3 \mathrm{~b}$ coated immune complexes are transported to lymph nodes and docked on follicular dendritic cells for long term antigen presentation important in the process of $B$ cell germinal center formation, and generation of antibodies [108]. By immunostaining of the popliteal lymph nodes, we evaluated the amount of phy- 
coerythrin transported to the B cell follicles in the presence of hC4Nb8 or a control nanobody. When hC4Nb8 was injected, the amount of antigen transported to the lymph nodes was significantly lower, as evaluated by the reduced fluorescence level of phycoerythrin colocalizing with follicular dendritic cells. Though hC4Nb8 was evaluated in a transgenic mouse model expressing human C4 alleles, the nanobody also binds to mouse C4b with an affinity of $0.2 \mathrm{nM}$ (Table 1), holding promise for its application in mouse models of disease linked to CP and LP overactivation. In the context of the CNS, hC4Nb8 was also shown to inhibit C3 deposition on neuronal cell cultures exposed to normal human serum as the source of complement [98]. In summary, the hC4Nb8 stands out as a powerful inhibitor of the complement cascade with an ultrahigh affinity for a neoepitope on C4b capable of interfering with complement activation through both the LP and CP. It is so far the only complement specific nanobody for which in vivo efficacy has been demonstrated, and it therefore appears useful for future work in animal disease models. Remarkably, despite very comprehensive efforts with respect to the development of complement inhibitors $[89,90]$, it represents the only antibody based C4b specific inhibitor described today. 


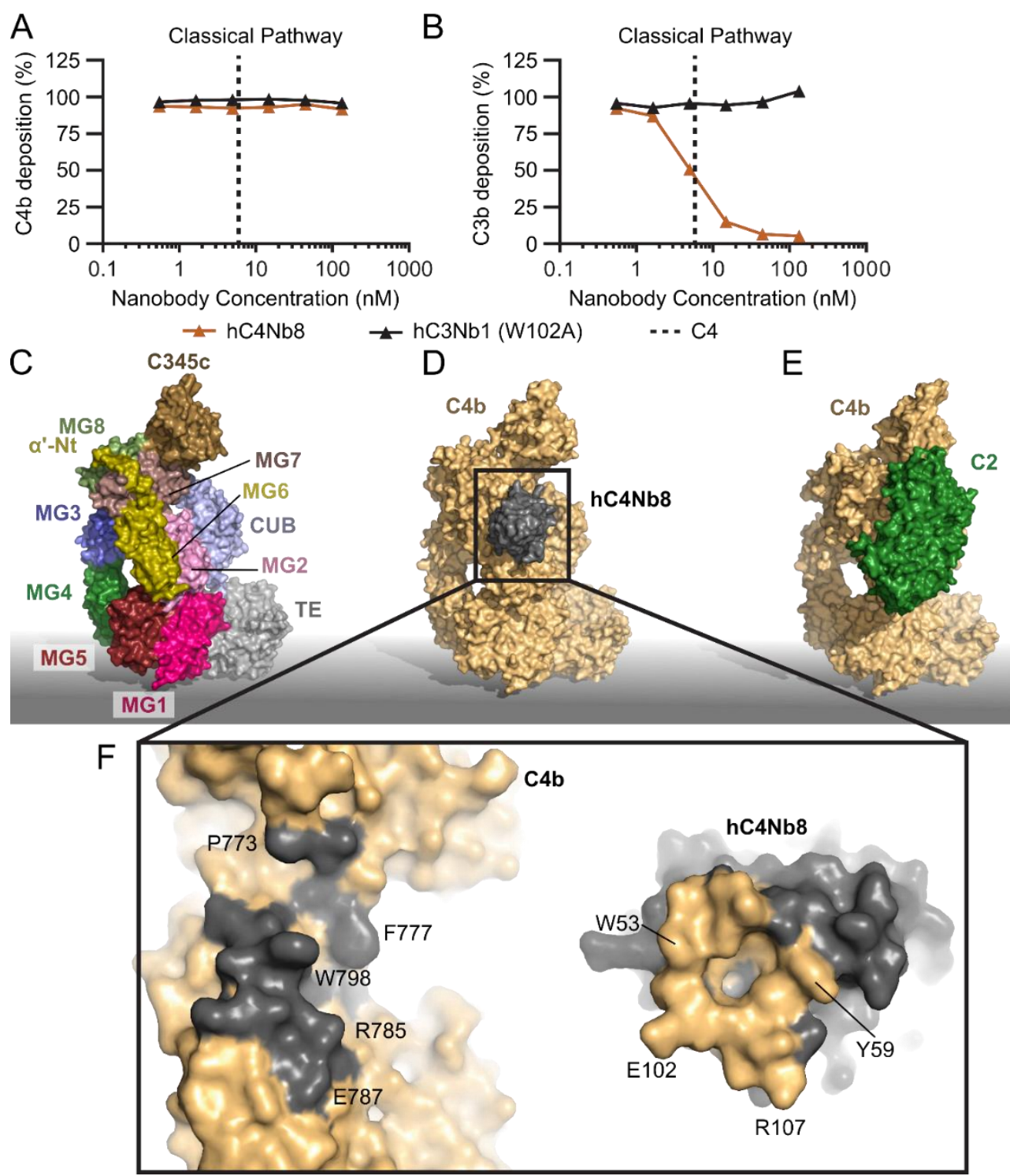

Figure 4. The inhibition mechanism of hC4Nb8. A-B) Effect of hC4Nb8 on C3b and C4b deposition in $0.2 \%$ NHS where the CP was activated by heat aggregated IgG. hC4Nb8 potently inhibits $\mathrm{C} 3 \mathrm{~b}$ deposition without interfering with $\mathrm{C} 4 \mathrm{~b}$ deposition. The inactive hC3Nb1 W102A is included as control. C) Domain structure of C4b [PDB entry 5JTW]. Labels indicate the location of the individual domains. D) the $\mathrm{C} 4 \mathrm{~b}: \mathrm{hC} 4 \mathrm{Nb} 8$ crystal structure [PDB entry 6YSQ]. E) the C4b2 atomic model based on SAXS data [106] highlights how hC4Nb8 binding to C4b prevents $\mathrm{C} 2$ from binding due to pronounced steric hindrance. F) An open book view of the hC4Nb8:C4b interface, revealing formation of a hydrophobic pocket in the hC4Nb8 paratope accommodating C4b Trp798. Important residues mediating the interaction are labeled. In panels D-F, C4b is shown in yellow, $\mathrm{hC} 4 \mathrm{Nb} 8$ in gray, and C2 in green.

\subsection{The hC3Nb1 is an inhibitor of AP proconvertase assembly}

Complement component $\mathrm{C} 3$ constitutes the point of convergence between the three pathways of the complement system, and C3 is a common target for complete inhibition of the complement cascade $[89,90]$. To date, we have described the three $\mathrm{C} 3$ specific nanobodies hC3Nb1 [99], hC3Nb2 [100] and hC3Nb3 [101]. Both hC3Nb1 and hC3Nb3 prevent the zymogen FB from binding to $\mathrm{C} 3 \mathrm{~b}$ by direct competition and inhibit $\mathrm{C} 3$ degradation through the alternative pathway. In contrast, $\mathrm{hC} 3 \mathrm{Nb} 2$ functions by preventing the substrate $\mathrm{C} 3$ from binding to either of the two $\mathrm{C} 3$ convertases, $\mathrm{C} 4 \mathrm{~b} 2 \mathrm{a}$ and $\mathrm{C} 3 \mathrm{bBb}$. 
We selected the first C3-specific nanobody, hC3Nb1, against human C3b, but the nanobody binds both native $\mathrm{C} 3$ and C3b strongly, with sub-nanomolar dissociation constants (Table 1). In complement deposition assays, the nanobody inhibits the progression of the AP mediated C3 fragment deposition (Figure 5A). In contrast, the nanobody did not inhibit C3 fragment deposition through the CP (Figure 5B) or CP mediated hemolysis of sheep erythrocytes (Figure 5C). As described above, C3 shares the overall domain structure with C4 (Figure 5D-E) and the crystal structures revealed that the hC3Nb1 nanobody binds at the interface between the MG6 and MG7 domains of native C3 and C3b [99, 109] (Figure 5F). By comparing the structure of hC3Nb1 in complex with $\mathrm{C} 3 \mathrm{~b}$ to the structure of the proconvertase $\mathrm{C} 3 \mathrm{bB}$ (Figure 5G) [31], we predicted that the nanobody inhibits the assembly of the alternative pathway C3 proconvertase through a steric clash with a glycan on FB. Through size exclusion chromatography, we showed that the presence of the nanobody indeed prevented C3bB assembly. Furthermore, when the glycan was eliminated from $\mathrm{FB}, \mathrm{hC} 3 \mathrm{Nb} 1$ no longer inhibited the assembly of the proconvertase.

Since hC3Nb1 was observed to bind tightly to both native $\mathrm{C} 3$ and $\mathrm{C} 3 \mathrm{~b}$, it was also investigated whether it may act at the level of $\mathrm{C} 3$ as a substrate for the $\mathrm{C} 3$ convertases. While the crystal structures of the both the AP C 3 convertase and the properdin-stabilized AP C3 convertase are known [30, 40, 110] (Figure 5H), structures of the substrate-bound convertases are not available, which complicates the prediction of how a C3 binding nanobody influences substrate recognition. However, we earlier proposed a general model for substrate-convertase interactions based on structural data [111]. This model states that the $\mathrm{C} 3$ convertases primarily recognize the substrate $\mathrm{C} 3$ through interactions with the $\mathrm{C} 3 \mathrm{~b}$ or $\mathrm{C} 4 \mathrm{~b}$ non-catalytic subunits, respectively, to position the scissile bond in the $\mathrm{C} 3$ substrate such that it can be cleaved by the catalytic subunits Bb and C2a in the AP and CP C3 convertase, respectively (Figure 5I). Specifically, the MG4 and MG5 domains in the C3 substrate are believed to interact with the MG4 and MG5 in the convertase C3b/C4b while the C3 substrate MG7 domain is suggested to be recognized by the MG6 and MG7 domains in the convertase $\mathrm{C} 3 \mathrm{~b} / \mathrm{C} 4 \mathrm{~b}$.

In the light of this model, the location of $\mathrm{hC} 3 \mathrm{Nb} 1$ on $\mathrm{C} 3$ suggested that the nanobody and $\mathrm{C} 3$ compete for binding to $\mathrm{C} 3$ as their binding sites overlap (Figure 5F vs Figure 5I). However, in the $\mathrm{CP}$ complement deposition assay, addition of hC3Nb1 conferred a nonsignificant effect, suggesting that the competition between hC3Nb1 and the CP C3 convertase is insufficient for inhibition of the CP C3 convertase or that the convertase-substrate model is imperfect. However, in accordance with this model hC3Nb1 did reduce the cleavage of $\mathrm{C} 3$ by the fluid phase AP C3 convertase CVF-Bb formed by cobra venom 
factor (CVF) and factor B [99]. CVF is a convenient reagent for analyzing C3 binding nanobodies as it allows uncoupling of the functions of C3 as a substrate and C3b as the noncatalytic subunit in the AP C3 convertase. These data obtained with the CP C3 convertase and CVF-Bb suggest that preventing the C3 substrate from binding to C3 convertases contributes modestly to the inhibition mode of hC3Nb1.

Importantly, it was also observed that hC3Nb1 interferes with the $\mathrm{FH}$ mediated cleavage of C3b by FI [99], which agrees with a predicted overlap between hC3Nb1 and the CCP1 domain of the FH cofactor (Figure 5J). The outcome of AP inhibition with hC3Nb1 in an in vivo setting will hence be the net result of these two opposing effects. On one hand, the nanobody will very efficiently inhibit the C3 turnover through the AP. On the other hand, the nanobody inhibits the endogenous regulation of the deposited C $3 \mathrm{~b}$ generated by the CP C3 convertase. As murine models are extensively used to study pathogenesis of complement associated diseases, we also investigated whether hC3Nb1 inhibits $\mathrm{C} 3$ fragment deposition in murine serum. Indeed, $\mathrm{hC} 3 \mathrm{Nb} 1$ does inhibit the $\mathrm{AP}$ in an assay conducted with murine serum, making it suitable for evaluation of AP inhibition in murine disease models. Lastly, our crystal structure allowed us to localize specific residues of the interaction interface between the nanobody and C3b (Figure 5K-L). This analysis allowed us to construct the inactive mutant of hC3Nb1 (W102A), which does not inhibit the AP (Figure 5A) and hC3Nb1 W102A is hence a perfect negative control for hC3Nb1. Overall, hC3Nb1 represents a high-affinity and cross-reactive C3 specific inhibitor of the alternative pathway mainly acting by preventing proconvertase assembly. 


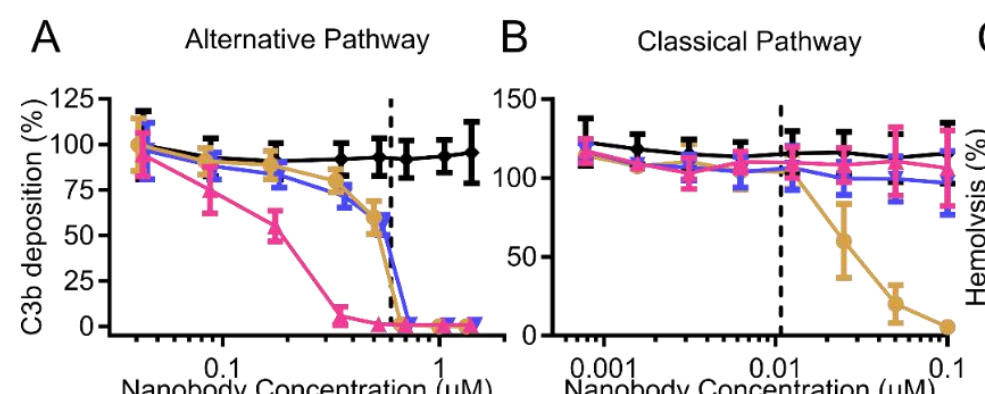

C Classical Pathway

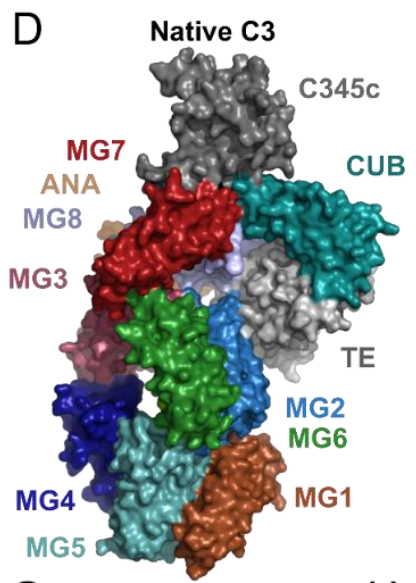

E

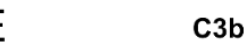

G
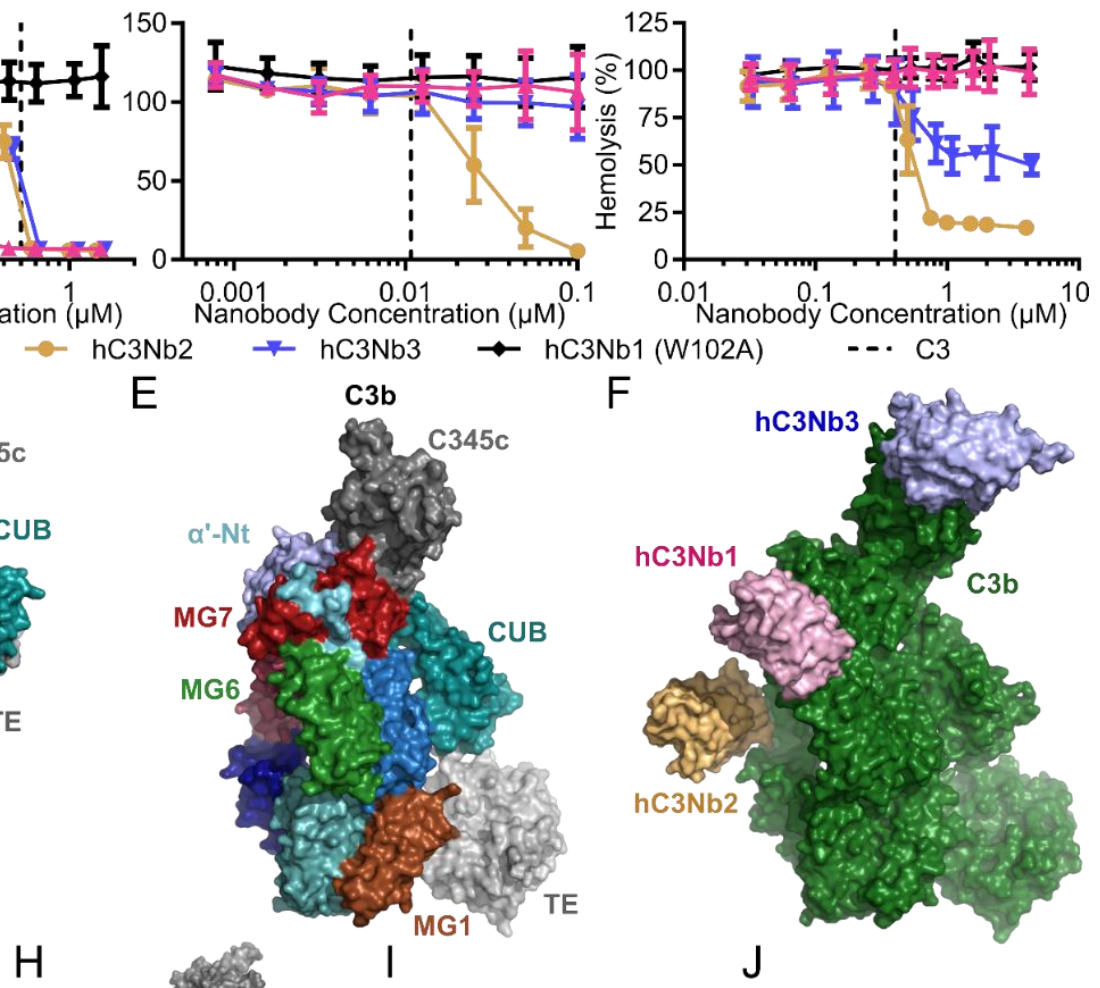

$\mathrm{F}$
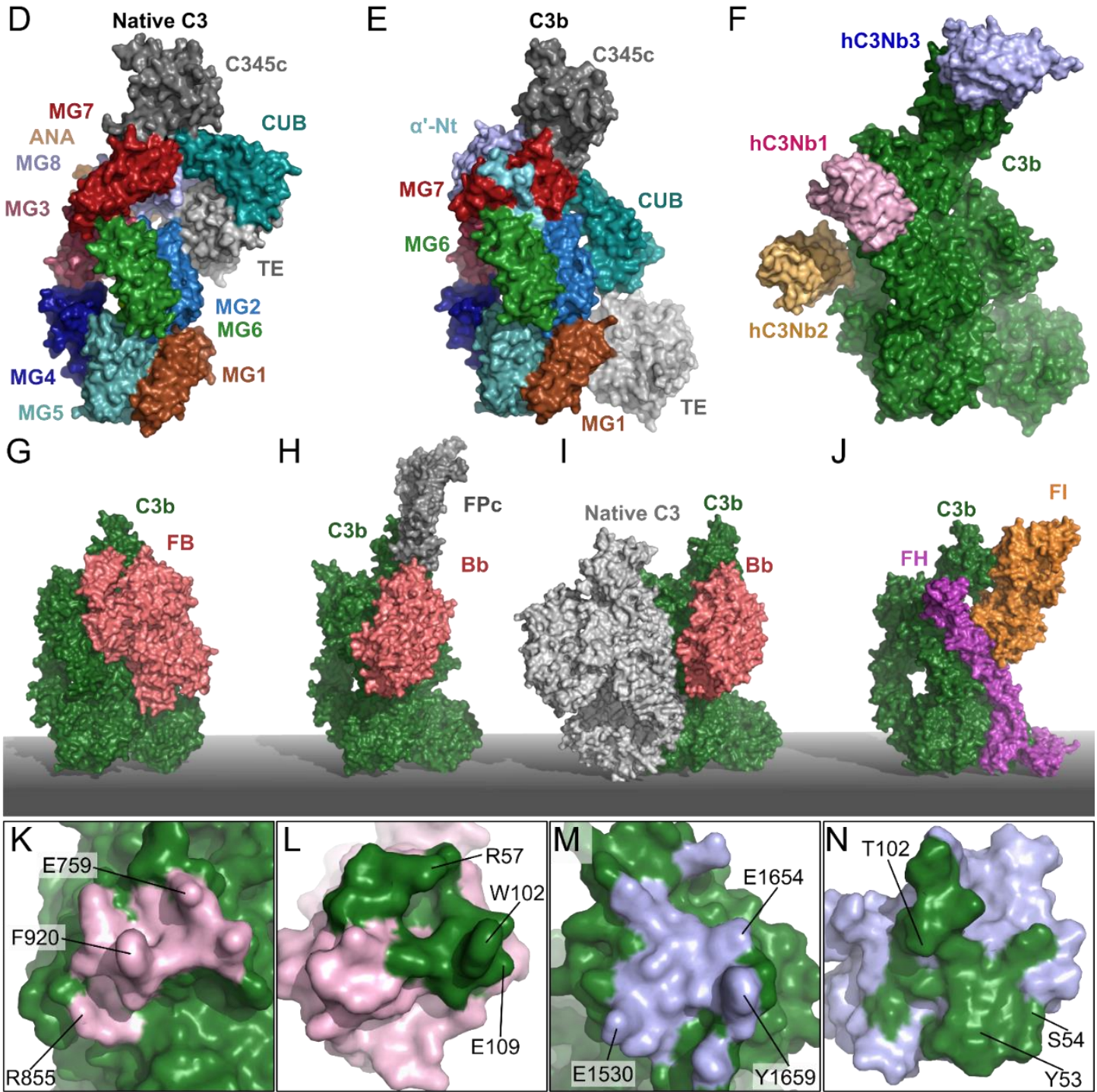

FI
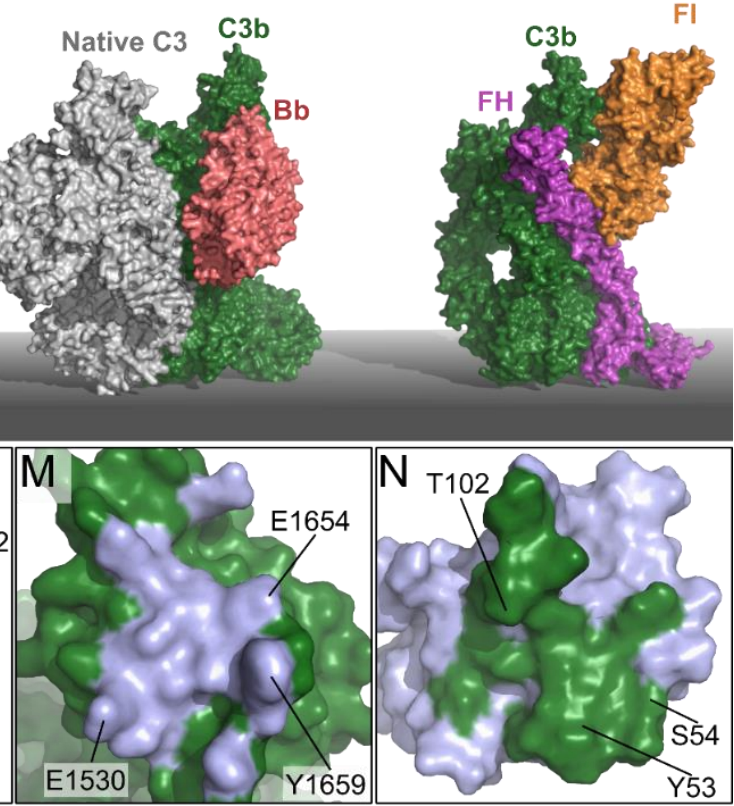

Figure 5. The C3 specific nanobodies. C3 fragment deposition assay elicited by (A) the AP in 11\% NHS or (B) the CP in $0.2 \%$ NHS demonstrating the effect of the $\mathrm{C} 3$ specific nanobodies: hC3Nb1 (AP inhibitor), hC3Nb2 (CP/LP/AP inhibitor), hC3Nb3 (AP inhibitor) as well as the inactive hC3Nb1 (W102A) mutant. (C) Classical pathway mediated hemolysis assay in 7.5\% NHS comparing the effects of hC3Nb1, hC3Nb2, hC3Nb3 and hC3Nb1 (W102A). Dashed lines in panels A-C indicate the putative C3 concentration in the assays. Surface representation of (D) native C3 [PDB entry 2A73] and (E) C3b [PDB entry 5FO7]. Labels indicate the location of the individual domains in panels D-E. (F) The proposed binding site of $\mathrm{hC} 3 \mathrm{Nb} 2$ as well as the crystal structure of the hC3Nb3 [PDB entry 6XZU] were docked onto the crystal structure of $\mathrm{hC} 3 \mathrm{Nb} 1$ in complex with $\mathrm{C} 3 \mathrm{~b}$ [6EHG]. Crystal structures of $(\mathrm{G})$ the AP C3 proconvertase [PDB entry: 2XWJ], and $(\mathrm{H})$ the properdin stabilized AP C3 convertase [PDB entry: 6RUR]. (I) The substrate recognition model of the C3 convertases, where C3 [PDB entry 
2A73] and C3bBb [PDB entry: 2WIN] were docked into the structure of the CVF:C5 complex [PDB entry: 3PVM]. (J) The crystal structure of the C3b:FI:mini-FH complex [PDB entry 5O32]. (K-L) Interaction sites indicated as footprints of $(\mathrm{K}) \mathrm{hC} 3 \mathrm{Nb} 1$ on $\mathrm{C} 3 \mathrm{~b},(\mathrm{~L}) \mathrm{C} 3 \mathrm{~b}$ on hC3Nb1, (M) hC3Nb3 on $\mathrm{C} 3 \mathrm{~b}$, and $(\mathrm{N}) \mathrm{C} 3 \mathrm{~b}$ on $\mathrm{hC} 3 \mathrm{Nb} 3$. Labels indicate selected residues in antigen and nanobody of importance for the interaction.

\section{$2.6 \mathrm{hC} 3 \mathrm{Nb} 2$ prevents substrate binding to $\mathrm{C} 3$ convertases}

The second C3 specific nanobody we characterized was hC3Nb2 [100], which was also selected using human C3b. C3 deposition assays revealed that the nanobody inhibits C3 deposition through all three pathways (Figure 5A-B), and furthermore inhibited C3 cleavage by the $\mathrm{CVFBb} \mathrm{C} 3$ convertase. This suggested that $\mathrm{hC} 3 \mathrm{Nb} 2$ acts on the substrate level to prevent the substrate from binding to the convertase and thereby inhibits $\mathrm{C} 3 \mathrm{deg}$ radation in contrast to hC3Nb1 that primarily acts by preventing proconvertase assembly. Deposition assays conducted with murine serum mirrored the results obtained in human serum showing that the nanobody inhibits both LP and AP mediated C3 fragment deposition in murine serum [100]. Accordingly, bio-layer interferometry analysis revealed similar dissociation constants of the nanobody for binding to murine and human C3b (Table 1) strengthening the notion that $\mathrm{hC} 3 \mathrm{Nb} 2$ is a cross-reactive complement inhibitor. Using negative stain electron microscopy, we mapped the epitope of the nanobody to the MG3 and MG4 domains (Figure 5F) of C3c, a stable degradation product of C3b. This binding site exhibits structural conservation amongst multiple functional states of $\mathrm{C} 3$ including native $\mathrm{C} 3, \mathrm{C} 3\left(\mathrm{H}_{2} \mathrm{O}\right), \mathrm{C} 3 \mathrm{~b}, \mathrm{iC} 3 \mathrm{~b}$ and the $\mathrm{C} 3 \mathrm{c}$ fragment used for mapping. This was confirmed by affinity measurements, where $\mathrm{hC} 3 \mathrm{Nb} 2$ bound the $\mathrm{C} 3\left(\mathrm{H}_{2} \mathrm{O}\right)$ analogue $\mathrm{C} 3$ methylamine (C3MA), $\mathrm{C} 3 \mathrm{~b}$ and native $\mathrm{C} 3$ with dissociation constants in the low nanomolar range (Table 1). Since the binding site is shared between $\mathrm{C} 3 \mathrm{c}$ and native $\mathrm{C} 3$, we could construct an overall model of the $\mathrm{C} 3-\mathrm{hC} 3 \mathrm{Nb} 2$ complex. Comparison of this with our general model of the convertase-substrate complexes [111, 112] (Figure 5I) confirmed that hC3Nb2 interferes with C3 substrate recognition by both C3 convertases C4b2a and $\mathrm{C} 3 \mathrm{bBb}$.

Lastly, we compared a model of the $\mathrm{C} 3 \mathrm{~b}-\mathrm{hC} 3 \mathrm{Nb} 2$ complex to the structure of the ternary complex between C3b, FI and FH [107] (Figure 5J). This comparison indicated that the $\mathrm{hC} 3 \mathrm{Nb} 2$ epitope lies far from the binding sites of FI and FH, and predicted that the nanobody in contrast to $\mathrm{hC} 3 \mathrm{Nb} 1$ does not interfere with endogenous regulation of the deposited C3b. Accordingly; a fluid phase assay revealed no effect of hC3Nb2 on the $\mathrm{FH}$ mediated cleavage of C3b by FI [100]. The cross-reactive hC3Nb2 nanobody thus inhibits all three pathways of the complement system, while it allows the degradation of $\mathrm{C} 3 \mathrm{~b}$ and it hence constitutes a potent inhibitor of the system. One disadvantage of the nanobody is 
however that native $\mathrm{C} 3$ normally present at seven $\mu \mathrm{M}$ in serum must be saturated, implying that $\mathrm{hC} 3 \mathrm{Nb} 2$ may need to be administrated such that its concentration remains at 0.1 $\mathrm{mg} / \mathrm{ml}$ if systemic C3 deposition is to be completely eliminated.

\section{7 hC3Nb3 provides insight into the role of $\mathrm{C} 3 \mathrm{~b}$ in $\mathrm{C} 5$ convertases}

The third C3-specific nanobody, hC3Nb3, was selected against the C345c domain of murine C3 from a library obtained from a llama immunized with human C3b. This selection strategy favored the selection of cross-reactive nanobodies that bind both human and murine $\mathrm{C} 3$. We demonstrated that $\mathrm{hC} 3 \mathrm{Nb} 3$ inhibits $\mathrm{C} 3$ deposition through the alternative pathway although less efficiently in murine serum as compared to human serum, where it efficiently blocks C3 fragment deposition (Figure 5A). Next, the complex between the nanobody and the antigen C 345 c domain of murine C 3 was crystallized, and the structure of the complex was determined to a maximum resolution of $1.5 \AA$ [101]. The structure revealed, that the nanobody grasps around the C-terminal helix of $\mathrm{C} 3 \mathrm{~b}$, through the formation of a concave binding site on the nanobody. Interestingly, the nanobody binds in a sideways manner and forms a binding site that involves extensive interactions between the framework region and the C-terminal helix of C3b (Figure 5M-N). Within the AP C3 proconvertase, $\mathrm{FB}$ interacts with the $\mathrm{C} 345 \mathrm{c}$ domain of $\mathrm{C} 3 \mathrm{~b}$, and by comparison of the crystal structure of the hC3Nb3:C345c complex with the structure of C3bB [31] (Figure 5G), we predicted that the nanobody would prevent the assembly of the alternative pathway C3 convertase, which was confirmed by size exclusion chromatography. In contrast, our structure also predicted that $\mathrm{hC} 3 \mathrm{Nb} 3$ does not prevent substrate $\mathrm{C} 3$ binding to the $\mathrm{CP} \mathrm{C} 3$ convertase or by the CVFBb $\mathrm{C} 3$ convertase, which was also confirmed experimentally (Figure 5B).

Based on the data presented above, hC3Nb3 appeared to be a highly specific AP inhibitor similar to hC3Nb1. However, deeper functional analyses showed that there are important differences between the two nanobodies. When projected onto $\mathrm{C} 3 \mathrm{~b}$, the hC3Nb3 epitope overlaps with the binding sites of AP regulators properdin (Figure $5 \mathrm{H}$ ) and factor I (Figure 5J). Accordingly, using bio-layer interferometry, we could confirm that in contrast to $\mathrm{hC} 3 \mathrm{Nb} 1, \mathrm{hC} 3 \mathrm{Nb} 3$ inhibits the interaction between $\mathrm{C} 3 \mathrm{~b}$ and properdin. Hence, in addition to preventing $\mathrm{C} 3 \mathrm{~b}$ binding to factor $\mathrm{B}, \mathrm{hC} 3 \mathrm{Nb} 3$ also prevents properdin from stabilizing the convertase. This further potentiates the inhibitory effect of the nanobody with respect to the AP as FP deficiency and depletion result in highly attenuated AP activity [110]. With respect to FI degradation of $\mathrm{C} 3 \mathrm{~b}$ to $\mathrm{iC} 3 \mathrm{~b}$, the in vitro cleavage assay somewhat surprisingly showed that hC3Nb3 only delayed the FH mediated cleavage of 
$\mathrm{C} 3 \mathrm{~b}$ by FI rather than completely preventing it. This observation suggests that the interaction between FI and the C3b C345c domain defined in the structure of the C3b:FH:FI complex [107] is not essential for FI recognition of the C3b:FH complex.

An additional intriguing observation was that $\mathrm{hC} 3 \mathrm{Nb} 3$ inhibited the formation of the C5b-9 complex upon activation of both $\mathrm{CP}$ and LP, which was unexpected since hC3Nb3 did not affect C3 deposition by the CP C3 convertase [5] (Figure 5B). To gain further insight into the apparent inhibition of the CP/LP C5 convertase, the effect of the nanobody on CP mediated lysis of sheep erythrocytes was tested. Similar to the C5b-9 deposition assays, hC3Nb3 efficiently reduced CP driven hemolysis [101] (Figure 5C). To exclude that this effect was due to residual AP activity, we also performed experiments in FB depleted serum that does not allow the assembly of the AP C3 convertase. This assay similarly showed a reduced hemolysis of sheep erythrocytes in the presence of hC3Nb3. As described above, buildup of the density of $\mathrm{C} 3 \mathrm{~b}$ on the activating surface induces a specificity shift in the two C3 convertases to function as C5 convertases. One explanation for the effect of $\mathrm{hC} 3 \mathrm{Nb} 3$ on the $\mathrm{CP}$ mediated hemolysis is hence, that the nanobody prevents the function of these auxiliary $\mathrm{C} 3 \mathrm{~b}$ molecules in the $\mathrm{C} 5$ convertase. As the AP C 3 convertase does not form efficiently in the presence of hC3Nb3, this effect could only be analyzed for the CP C 5 convertase. Whether the nanobody directly inhibits the $\mathrm{C} 5$ substrate recognition or rather the assembly of the CP C5 convertase is difficult to determine as the exact role of $\mathrm{C} 3 \mathrm{~b}$ in the CP C5 convertase remains unsettled [112]. In summary, hC3Nb3 is a highaffinity AP inhibitor preventing assembly of the proconvertase without interfering strongly with FI degradation of C3b. Through its binding to C3b, hC3Nb3 also interferes with the substrate cleavage by the CP C5 convertase through a yet unknown mechanism.

\subsection{The non-inhibitory properdin specific hFPNb1}

Whereas the C1q, C4 and C3 specific nanobodies described above are inhibitory and therefore candidates for complement therapeutics, the properdin specific nanobody hFPNb1 offers a demonstration of how non-inhibitory nanobodies can support structurefunction studies of complex molecular pathways like the complement cascade. As alluded to above, properdin circulates in three oligomeric states in circulation and adopts very extended structures [113]. To tackle its structure determination, Pedersen et al generated a monomeric version of properdin, named FPc [109]. In addition, Pedersen et al generated a library and selected nanobodies against properdin, in order to isolate nanobody binders that could promote structure determination of the complex between the monomeric properdin and the AP C3 convertase $\mathrm{C} 3 \mathrm{bBb}$. The hFPNb1 nanobody was crystallized in com- 
plex with C3bBb:FPc and the staphylococcal complement inhibitor SCIN at $6.2 \AA$ resolution [40]. The structure revealed its epitope located on FPc TSR4, far from C3b and Bb, in accordance with absence of AP inhibitory activity of this nanobody. The study underlines the well-known potential of nanobodies as crystallization chaperones that can trap macromolecules in a specific conformation, enabling crystallization of challenging targets including membrane proteins and their complexes.

\subsection{Vsig4 specific nanobodies}

In addition to the nanobodies described above, a nanobody targeting the Vsig4 receptor (also known as complement receptor Ig, (CRIg) has also been characterized [102]. Besides being involved in regulation of $\mathrm{T}$ cell immunity, Vsig4 is also a complement receptor expressed exclusively on tissue resident macrophages, where it mediates the clearance of circulating complement-opsonized cells, through recognition of the MG4 and MG5 domains of $\mathrm{C} 3 \mathrm{~b}$ and $\mathrm{iC} 3 \mathrm{~b}[48,114]$. The ectodomain of Vsig4 is a possible therapeutic molecule that through binding to $\mathrm{C} 3 \mathrm{~b}$ and $\mathrm{iC} 3 \mathrm{~b}$ may control diseases associated to excessive $\mathrm{CP}$ and AP activation [115]. Vsig4 specific nanobodies were selected from a library obtained after immunization of an alpaca with the extracellular domain of murine Vsig4. The nanobody Nb119 binds to the binding site of the C3b MG4 and MG5 domains on Vsig4, thereby preventing the endogenous interaction [102]. The nanobody is cross reactive with human Vsig4, albeit with a 250-fold lower affinity. The crystal structures of the complexes of the $\mathrm{Nb119}$ with human and murine Vsig4 demonstrated that the decreased affinity for hVsig4 is due to changes in CDR3 of Nb119. Single residue mutations guided by the available atomic models could be introduced for improving the $\mathrm{Nb} 119$ affinity toward hVsig4 [102].

\section{Perspectives}

\subsection{Complement specific nanobodies as tools in diagnostics and research}

Due to their single domain nature, nanobodies are relatively easy to engineer and multiple examples of insertion of site-specific modifications or fusion proteins have been described in the literature. These include addition of fluorophores and radioligands, and fusion to Fc-fragments or fluorescent proteins. For live imaging, nanobodies are superior to monoclonal antibodies, due to their shorter circulation time, which results in a decreased background signal. An interesting feature of the hC3Nb3 nanobody is its very strong and SDS-resistant interaction with the C345c domain of C3. We frequently observed the formation of SDS-PAGE resistant complexes between the nanobody and the 
various functional states of $\mathrm{C} 3$, which was used to assay the relative amount of the individual C3 degradation products in samples [101]. This also led us to explore other applications of the nanobody. Through fusion to an IgY Fc fragment, we produced the nanobody into an antibody format and directly conjugated a fluorophore to the fusion protein. Using this hC3Nb3-IgY Fc fusion protein, we performed immunohistochemistry of C3 deposited on in vitro differentiated neurons. This staining revealed C3 deposition on a subset of the neurites [101]. In an orthogonal approach, C3 fragments on pig erythrocytes were quantitated by flow cytometry using a hC3Nb3-GFP fusion protein as the marker [101]. These properties demonstrate the potential of using the hC3Nb3 nanobody in diagnostic applications quantitating C3 deposition. An additional example is offered by the Vsig4 specific nanobody Nb119 labeled with 99mTc [116]. This allows imaging of inflamed joints in collagen induced arthritis (CIA) mice, a model that mimics the human rheumatoid arthritis immunologic state [117]. The accumulation of ${ }^{99 \mathrm{~m} T c-N b 119}$ in CIA mice joints correlated well with disease severity assigned by the macroscopic arthritis scoring system, but the nanobody also allowed detection of knee inflammation prior to onset of macroscopic symptoms [116]. The results from this study indicate that a similar approach could be followed to diagnose human rheumatoid arthritis even before macroscopic detection of joint inflammation is possible. The authors further characterized the ability of ${ }^{99 \mathrm{mTc}} \mathrm{T}$ $\mathrm{Nb} 119$ to follow the stages of disease development by using STIA mice, where arthritis is temporarily induced by transfer of arthritogenic serum and resolves after 15-30 days [118]. In this way, Zheng et al showed that ${ }^{99 \mathrm{~m} T c-N b 119}$ signal correlates with the stage of disease progression, demonstrating its applicability to monitor disease evolution in a non-invasive manner [119].

Complement specific nanobodies have also demonstrated to be valuable reagents for basic research aiming at analyzing the contribution of the individual pathways to activation of the cascade. Anti- $\alpha \mathrm{Gal}$ antibodies were thought to inhibit complement on pathogen surfaces, nonetheless these antibodies react with a number of pathogens [120]. Jensen et al recently utilized $\mathrm{hC} 3 \mathrm{Nb} 1, \mathrm{hC} 3 \mathrm{Nb} 2$, and $\mathrm{C} 1 \mathrm{qNb} 75$ to attribute the complement pathways elicited by these antibodies targeting the galactose- $\alpha-1,3$-galactose carbohydrate. The authors reported that the anti- $\alpha$ Gal antibodies elicit complement deposition by activating the classical pathway [121]. This basic research application is an example of how complement inhibitory nanobodies can be used to assign the contribution of the individual pathways to complement activation. Recently, Lausen et al utilized hC3Nb2 to broadly inhibit the in vitro complement deposition on Chlamydia trachomatis and demonstrated complement dependent adherence of the bacteria to B-cells [122]. 


\subsection{Nanobody driven activation of complement}

Bispecific monoclonal antibodies that target bacterial surface proteins or $\mathrm{B}$ and $\mathrm{T}$ cell antigens with one arm and $\mathrm{C} 1 \mathrm{q}$ with the other arm were previously investigated as a means of targeting complement to specific cell types. It was demonstrated that such bifunctional antibodies could elicit complement dependent cytotoxicity on different types of cells [103]. The potential of complement targeting by nanobody fusion proteins was explored by Pedersen et al, who directed complement activation to tumor cells by combining $\mathrm{hFPNb} 1$ with a nanobody targeting the epidermal growth factor receptor expressed on cancer cells [123]. Although C3 deposition could be detected on the cells targeted, this did not result in complement dependent cytotoxicity [123]. It was not possible to conclude whether this was due to absence of $\mathrm{C} 5$ convertase formation or due to a high abundance of complement regulators on the surface of cancer cells. Nevertheless, the demonstration that nanobody driven targeting of properdin supports AP initiation offers a tool to future experiments aiming at exploring the somewhat controversial concept that properdin can act as $\mathrm{C} 3 \mathrm{~b}$ independent pattern recognition molecule capable of initiating the AP pathway on a surface without prior C3 deposition [36].

\section{Conclusions}

The complement system is now recognized to be involved in the pathogenesis of numerous diseases and consequently inhibiting complement is gaining increased attention. Currently, the only FDA approved complement specific inhibitors are the anti-C5 monoclonal antibody eculizumab and its derivative ravulizumab (Alexion pharmaceuticals). Both antibodies inhibit C5 cleavage and initiation of the terminal complement pathway. Eculizumab is indicated for treatment of patients with PNH, aHUS and generalized myasthenia gravis while ravulizumab is approved for treatment of patients with $\mathrm{PNH}$ and aHUS. However, with the general acceptance of complement proteins as validated therapeutic targets, many other complement modulating compounds are now in development $[89,124]$. These include monoclonal antibodies, peptides and small molecules, but complement specific nanobodies thus far represent an underexplored modality for complement inhibition. We foresee an increased interest in developing complement specific nanobodies due to their unique characteristicof binding with high affinity and specificity to their target antigen combined with their single domain structure, allowing easy protein engineering. While unmodified nanobodies are rapidly cleared by renal filtration, different half-life extension strategies exists including addition of PEG, fusion of the nanobody to an IgG framework, or conjugation to an albumin binding molecule [125]. Furthermore, complement inhibiting nanobodies can be targeted to specific tissues to avoid systemic 
inhibition in analogy to what has been shown with the complement inhibitor B4Crry [126]. We have presented seven nanobodies that bind different complement components and outlined the biochemical and structural characterization of their interaction with their respective antigens. Besides the inhibitory activity of the nanobodies described in the first part, we discuss potential applications of the nanobodies for detecting complement deposition on cells or for bioimaging of joint inflammation. As basic research tools, nanobodies were shown to mediate targeting of complement to a surface, and to allow unambiguous assignment of the complement activating ability of anti- $\alpha$ Gal IgGs. These are a few examples of the opportunities offered by the complement specific nanobodies presented. Further research could identify their potential in transient complement inhibition instead of complement gene knockout in animal models, thus avoiding associated side effects, as development of SLE and auto-reactive B cells for $\mathrm{C} 1 \mathrm{q} \mathrm{KO}$ and C4 KO mice respectively $[76,127]$. The inhibitory nanobodies may also be administrated in the central nervous system in animal models of schizophrenia, to evaluate the effect of $\mathrm{C} 3$ convertase inhibition and their potential for therapeutic applications in the treatment of this and other neurodegenerative diseases.

Author Contributions: All authors wrote, read and agreed to the published version of the manuscript.

Funding: This research was funded by the Lundbeck Foundation (BRAINSTRUC, grant no. R1552015-2666), the Novo Nordisk Foundation (grant no NNF16OC0022058), the Lundbeck Foundation grant no. R192-2015-726.

Institutional Review Board Statement: Not applicable.

Informed Consent Statement: Not applicable.

Acknowledgments: Our experimental work with nanobodies was supported by excellent technical assistance from Karen Magrethe Nielsen. We also acknowledge the long term collaboration with the research team of Steffen Thiel and their contributions to our development and functional evaluation of complement specific nanobodies .

Conflicts of Interest: GRA and NSL declare collaboration with Alexion Pharmaceuticals. All authors are inventors on patent WO2019/238674A1 describing C1qNb75, hC4Nb8, hC3Nb2 and hC3Nb3.

\section{References}

1. Sharp, T.H., et al., Insights into IgM-mediated complement activation based on in situ structures of IgM-C1-C4b. Proceedings of the National Academy of Sciences, 2019. 116(24): p. 11900-11905.

2. Hajishengallis, G., et al., Novel mechanisms and functions of complement. Nat Immunol, 2017. 18(12): p. 1288-1298. 
3. Merle, N.S., et al., Complement System Part I - Molecular Mechanisms of Activation and Regulation. Front Immunol, 2015. 6: p. 262.

4. Bajic, G., et al., Complement activation, regulation, and molecular basis for complement-related diseases. The EMBO Journal, 2015. 34(22): p. 2735-2757.

5. Laursen, N.S., et al., Functional and Structural Characterization of a Potent C1q Inhibitor Targeting the Classical Pathway of the Complement System. Frontiers in Immunology, 2020. 11(1504).

6. Janeway, C.A., Pillars Article: Approaching the Asymptote? Evolution and Revolution in Immunology. $<$ em $>$ Cold Spring Harb Symp Quant Biol</em>. 1989. 54: 1-13. The Journal of Immunology, 2013. 191(9): p. 4475-4487.

7. Roumenina, L.T., et al., Interaction of C1q with IgG1, C-reactive protein and pentraxin 3: mutational studies using recombinant globular head modules of human C1q A, B, and C chains. Biochemistry, 2006. 45(13): p. 4093-4104.

8. $\quad$ Païdassi, H., et al., C1q Binds Phosphatidylserine and Likely Acts as a Multiligand-Bridging Molecule in Apoptotic Cell Recognition. The Journal of Immunology, 2008. 180(4): p. 2329-2338.

9. Gaboriaud, C., et al., The human c1q globular domain: structure and recognition of non-immune self ligands. Front Immunol, 2011. 2: p. 92.

10. Tacnet-Delorme, P., S. Chevallier, and G.J. Arlaud, $\beta$-Amyloid Fibrils Activate the C1 Complex of Complement Under Physiological Conditions: Evidence for a Binding Site for A $\beta$ on the C1q Globular Regions. The Journal of Immunology, 2001. 167(11): p. 6374-6381.

11. Erlich, P., et al., Complement protein C1q forms a complex with cytotoxic prion protein oligomers. The Journal of biological chemistry, 2010. 285(25): p. 19267-19276.

12. Ugurlar, D., et al., Structures of C1-IgG1 provide insights into how danger pattern recognition activates complement. Science, 2018. 359(6377): p. 794-797.

13. Bally, I., et al., Interaction of C1q With Pentraxin 3 and IgM Revisited: Mutational Studies With Recombinant C1q Variants. Frontiers in Immunology, 2019. 10(461).

14. Zlatarova, A.S., et al., Existence of Different but Overlapping IgG- and IgM-Binding Sites on the Globular Domain of Human C1q. Biochemistry, 2006. 45(33): p. 9979-9988.

15. Diebolder, C.A., et al., Complement is activated by IgG hexamers assembled at the cell surface. Science (New York, N.Y.), 2014. 343(6176): p. 1260-1263.

16. Kidmose, R.T., et al., Structural basis for activation of the complement system by component C4 cleavage. Proceedings of the National Academy of Sciences, 2012. 109(38): p. 15425-15430.

17. Mortensen, S., et al., Structural Basis for the Function of Complement Component C4 within the Classical and Lectin Pathways of Complement. The Journal of Immunology, 2015. 194(11): p. 5488-5496.

18. Sitomer, G., R.M. Stroud, and M.M. Mayer, Reversible adsorption of C'2 by EAC'4: Role of Mg2+, enumeration of competent $S A C^{\prime} 4$, two-step nature of $C^{\prime} 2 a$ fixation and estimation of its efficiency. Immunochemistry, 1966. 3(1): p. 57-69.

19. Müller-Eberhard, H.J., M.J. Polley, and M.A. Calcott, Formation and functional significance of a molecular complex derived from the second and the fourth component of human complement. J Exp Med, 1967. 125(2): p. 359-80.

20. Wallis, R., et al., Molecular Interactions between MASP-2, C4, and C2 and Their Activation Fragments Leading to Complement Activation via the Lectin Pathway. Journal of Biological Chemistry, 2007. 282(11): p. 7844-7851.

21. Gadjeva, M., S. Thiel, and J.C. Jensenius, The mannan-binding-lectin pathway of the innate immune response. Current Opinion in Immunology, 2001. 13(1): p. 74-78.

22. Matsushita, M. and T. Fujita, Ficolins and the lectin complement pathway. Immunological Reviews, 2001. 180(1): p. 78-85.

23. Henriksen, M.L., et al., Heteromeric Complexes of Native Collectin Kidney 1 and Collectin Liver 1 Are Found in the Circulation with MASPs and Activate the Complement System. The Journal of Immunology, 2013. 191(12): p. 6117-6127.

24. Matsushita, M. and T. Fujita, Activation of the classical complement pathway by mannose-binding protein in association with a novel C1s-like serine protease. The Journal of Experimental Medicine, 1992. 176(6): p. 1497-1502. 
25. Thiel, S., et al., A second serine protease associated with mannan-binding lectin that activates complement. Nature, 1997. 386(6624): p. 506-510.

26. Dahl, M.R., et al., Mannan-binding lectin associated serine protease 3 (MASP-3) - a new component of the lectin pathway of complement activation. Immunopharmacology, 2000. 49(1): p. 79.

27. Janssen, B.J.C., et al., Structures of complement component C3 provide insights into the function and evolution of immunity. Nature, 2005. 437(7058): p. 505-511.

28. Janssen, B.J.C., et al., Structure of C3b reveals conformational changes that underlie complement activity. Nature, 2006. 444(7116): p. 213-216.

29. Pangburn, M.K. and H.J. Müller-Eberhard, The C3 convertase of the alternative pathway of human complement. Enzymic properties of the bimolecular proteinase. The Biochemical journal, 1986. 235(3): p. 723-730.

30. Rooijakkers, S.H.M., et al., Structural and functional implications of the alternative complement pathway C3 convertase stabilized by a staphylococcal inhibitor. Nature Immunology, 2009. 10: p. 721.

31. Forneris, F., et al., Structures of C3b in complex with factors B and D give insight into complement convertase formation. Science (New York, N.Y.), 2010. 330(6012): p. 1816-1820.

32. Lachmann, P.J., Chapter 4 - The Amplification Loop of the Complement Pathways, in Advances in Immunology, F.W. Alt, Editor. 2009, Academic Press. p. 115-149.

33. Harboe, M., et al., The down-stream effects of mannan-induced lectin complement pathway activation depend quantitatively on alternative pathway amplification. Mol Immunol, 2009. 47(2-3): p. 373-80.

34. Harboe, M., et al., The quantitative role of alternative pathway amplification in classical pathway induced terminal complement activation. Clin Exp Immunol, 2004. 138(3): p. 439-46.

35. Fishelson, Z., M.K. Pangburn, and H.J. Müller-Eberhard, Characterization of the initial C3 convertase of the alternative pathway of human complement. The Journal of Immunology, 1984. 132(3): p. 1430-1434.

36. Harrison, R.A., The properdin pathway: an "alternative activation pathway" or a "critical amplification loop" for C3 and C5 activation? Semin Immunopathol, 2018. 40(1): p. 15-35.

37. Ekdahl, K.N., et al., Is generation of C3(H2O) necessary for activation of the alternative pathway in real life? Molecular Immunology, 2019. 114: p. 353-361.

38. Fearon, D.T. and K.F. Austen, Properdin: binding to C3b and stabilization of the C3b-dependent C3 convertase. The Journal of Experimental Medicine, 1975. 142(4): p. 856-863.

39. Hourcade, D.E., The Role of Properdin in the Assembly of the Alternative Pathway C3 Convertases of Complement. Journal of Biological Chemistry, 2006. 281(4): p. 2128-2132.

40. Pedersen, D.V., et al., Structural Basis for Properdin Oligomerization and Convertase Stimulation in the Human Complement System. Frontiers in Immunology, 2019. 10(2007).

41. Hourcade, D., V.M. Holers, and J.P. Atkinson, The Regulators of Complement Activation (RCA) Gene Cluster, in Advances in Immunology, F.J. Dixon, Editor. 1989, Academic Press. p. 381-416.

42. Liszewski, M.K., et al., Dissecting sites important for complement regulatory activity in membrane cofactor protein (MCP; CD46). J Biol Chem, 2000. 275(48): p. 37692-701.

43. Krych-Goldberg, M. and J.P. Atkinson, Structure-function relationships of complement receptor type 1. Immunol Rev, 2001. 180: p. 112-22.

44. Jozsi, M. and P.F. Zipfel, Factor H family proteins and human diseases. Trends Immunol, 2008. 29(8): p. 380-7.

45. $\mathrm{Wu}, \mathrm{J}$. , et al., Structure of complement fragment C3b-factor $\mathrm{H}$ and implications for host protection by complement regulators. Nature immunology, 2009. 10(7): p. 728-733.

46. Ricklin, D., E.S. Reis, and J.D. Lambris, Complement in disease: a defence system turning offensive. Nature reviews. Nephrology, 2016. 12(7): p. 383-401. 
47. Coulthard, L.G. and T.M. Woodruff, Is the Complement Activation Product C3a a Proinflammatory Molecule? Re-evaluating the Evidence and the Myth. The Journal of Immunology, 2015. 194(8): p. 3542-3548.

48. Helmy, K.Y., et al., CRIg: a macrophage complement receptor required for phagocytosis of circulating pathogens. Cell, 2006. 124(5): p. 915-27.

49. Liu, G., et al., CRIg plays an essential role in intravascular clearance of bloodborne parasites by interacting with complement. Proc Natl Acad Sci U S A, 2019. 116(48): p. 24214-24220.

50. Vorup-Jensen, T. and R.K. Jensen, Structural Immunology of Complement Receptors 3 and 4. Front Immunol, 2018.9 : p. 2716.

51. Gonzalez, S.F., et al., Complement-Dependent Transport of Antigen into B Cell Follicles. The Journal of Immunology, 2010. 185(5): p. 2659-2664.

Pangburn, M.K. and N. Rawal, Structure and function of complement C5 convertase enzymes. Biochemical Society Transactions, 2002. 30(6): p. 1006-1010.

Rawal, N. and M.K. Pangburn, Formation of High Affinity C5 Convertase of the Classical Pathway of Complement. Journal of Biological Chemistry, 2003. 278(40): p. 38476-38483.

Fredslund, F., et al., Structure of and influence of a tick complement inhibitor on human complement component 5. Nat Immunol, 2008. 9(7): p. 753-60.

Bayly-Jones, C., D. Bubeck, and M.A. Dunstone, The mystery behind membrane insertion: a review of the complement membrane attack complex. Philosophical transactions of the Royal Society of London. Series B, Biological sciences, 2017. 372(1726): p. 20160221.

56. Heesterbeek, D.A.C., et al., Complement and Bacterial Infections: From Molecular Mechanisms to Therapeutic Applications. Journal of Innate Immunity, 2018. 10(5-6): p. 455-464.

57. Klos, A., et al., The role of the anaphylatoxins in health and disease. Molecular Immunology, 2009. 46(14): p. $2753-2766$.

58. Hill, A., et al., Paroxysmal nocturnal haemoglobinuria. Nature reviews. Disease primers, 2017. 3: p. 17028-17028.

59. Nicholson-Weller, A., et al., Affected erythrocytes of patients with paroxysmal nocturnal hemoglobinuria are deficient in the complement regulatory protein, decay accelerating factor. Proc Natl Acad Sci U S A, 1983. 80(16): p. 5066-70.

60. Holguin, M.H., et al., Isolation and characterization of a membrane protein from normal human erythrocytes that inhibits reactive lysis of the erythrocytes of paroxysmal nocturnal hemoglobinuria. J Clin Invest, 1989. 84(1): p. 7-17.

61. Zhang, K., et al., Atypical Hemolytic Uremic Syndrome: A Brief Review. Hematology reports, 2017. 9(2): p. $7053-7053$.

62. Bresin, E., et al., Combined complement gene mutations in atypical hemolytic uremic syndrome influence clinical phenotype. Journal of the American Society of Nephrology : JASN, 2013. 24(3): p. 475-486.

63. Rodríguez de Córdoba, S., et al., Genetics of atypical hemolytic uremic syndrome (aHUS). Seminars in thrombosis and hemostasis, 2014. 40(4): p. 422-430.

64. Fakhouri, F., et al., C3 glomerulopathy: a new classification. Nat Rev Nephrol, 2010. 6(8): p. $494-9$.

65. Smith, R.J.H., et al., C3 glomerulopathy - understanding a rare complement-driven renal disease. Nature reviews. Nephrology, 2019. 15(3): p. 129-143.

66. Zhang, Y., et al., Causes of alternative pathway dysregulation in dense deposit disease. Clin J Am Soc Nephrol, 2012. 7(2): p. 26574.

67. Bu, F., et al., High-Throughput Genetic Testing for Thrombotic Microangiopathies and C3 Glomerulopathies. Journal of the American Society of Nephrology, 2016. 27(4): p. 1245-1253.

68. van Lookeren Campagne, M., E.C. Strauss, and B.L. Yaspan, Age-related macular degeneration: Complement in action. Immunobiology, 2016. 221(6): p. 733-739.

69. Fritsche, L.G., et al., Seven new loci associated with age-related macular degeneration. Nat Genet, 2013. 45(4): p. 433-9, 439e1-2.

70. Ogden, C.A., et al., C1q and mannose binding lectin engagement of cell surface calreticulin and CD91 initiates macropinocytosis and uptake of apoptotic cells. The Journal of experimental medicine, 2001. 194(6): p. 781-795.

71. Botto, M. and M.J. Walport, C1q, Autoimmunity and Apoptosis. Immunobiology, 2002. 205(4): p. 395-406. 
72. Garcia, B.L., et al., Novel Evasion Mechanisms of the Classical Complement Pathway. Journal of immunology (Baltimore, Md. : 1950), 2016. 197(6): p. 2051-2060.

73. Jäger, U., et al., Inhibition of complement C1s improves severe hemolytic anemia in cold agglutinin disease: a first-in-human trial. Blood, 2019. 133(9): p. 893-901.

74. Ricklin, D. and J.D. Lambris, Complement in Immune and Inflammatory Disorders: Therapeutic Interventions. The Journal of Immunology, 2013. 190(8): p. 3839.

75. Wouters, D. and S. Zeerleder, Complement inhibitors to treat IgM-mediated autoimmune hemolysis. Haematologica, 2015. 100(11): p. 1388-1395.

76. Lintner, K.E., et al., Early Components of the Complement Classical Activation Pathway in Human Systemic Autoimmune Diseases. Frontiers in Immunology, 2016. 7(36).

77. Johnson, M.B. and B. Stevens, Pruning hypothesis comes of age. Nature, 2018. 554(7693): p. 438-439.

78. Stevens, B., et al., The Classical Complement Cascade Mediates CNS Synapse Elimination. Cell, 2007. 131(6): p. 1164-1178.

79. Stephan, A.H., B.A. Barres, and B. Stevens, The Complement System: An Unexpected Role in Synaptic Pruning During Development and Disease. Annual Review of Neuroscience, 2012. 35(1): p. 369-389.

80. Sekar, A., et al., Schizophrenia risk from complex variation of complement component 4. Nature, 2016. 530(7589): p. 177-183.

81. Schizophrenia Working Group of the Psychiatric Genomics, C., Biological insights from 108 schizophrenia-associated genetic loci. Nature, 2014. 511(7510): p. 421-427.

82. Baum, M.L., et al., CUB and Sushi Multiple Domains 1 (CSMD1) opposes the complement cascade in neural tissues. bioRxiv, 2020: p. 2020.09.11.291427.

83. Comer, A.L., et al., Increased expression of schizophrenia-associated gene C4 leads to hypoconnectivity of prefrontal cortex and reduced social interaction. PLOS Biology, 2020. 18(1): p. e3000604.

84. Lui, H., et al., Progranulin Deficiency Promotes Circuit-Specific Synaptic Pruning by Microglia via Complement Activation. Cell, 2016. 165(4): p. 921-935.

85. Hong, S., et al., Complement and microglia mediate early synapse loss in Alzheimer mouse models. Science, 2016. 352(6286): p. 712-716.

86. Vukojicic, A., et al., The Classical Complement Pathway Mediates Microglia-Dependent Remodeling of Spinal Motor Circuits during Development and in SMA. Cell Reports, 2019. 29(10): p. 3087-3100.e7.

87. Carpanini, S.M., M. Torvell, and B.P. Morgan, Therapeutic Inhibition of the Complement System in Diseases of the Central Nervous System. Frontiers in immunology, 2019. 10: p. 362-362.

88. Vasek, M.J., et al., A complement-microglial axis drives synapse loss during virus-induced memory impairment. Nature, 2016. 534: p. 538.

89. Zelek, W.M., et al., Compendium of current complement therapeutics. Mol Immunol, 2019. 114: p. 341-352.

90. Ricklin, D., et al., The renaissance of complement therapeutics. Nat Rev Nephrol, 2018. 14(1): p. $26-47$.

91. Mohebnasab, M., et al., Current and Future Approaches for Monitoring Responses to Anti-complement Therapeutics. Frontiers in Immunology, 2019. 10(2539).

92. Muyldermans, S., Nanobodies: natural single-domain antibodies. Annu Rev Biochem, 2013. 82: p. 775-97.

93. Pardon, E., et al., A general protocol for the generation of Nanobodies for structural biology. Nature protocols, 2014. 9(3): p. 674693.

94. Moller-Kristensen, M., et al., On the site of C4 deposition upon complement activation via the mannan-binding lectin pathway or the classical pathway. Scand J Immunol, 2003. 57(6): p. 556-61.

95. Frederiksen, P.D., et al., Quantification of mannan-binding lectin. J Immunol Methods, 2006. 315(1-2): p. 49-60.

96. Petersen, S.V., et al., An assay for the mannan-binding lectin pathway of complement activation. J Immunol Methods, 2001. 257(1-2): p. 107-16. 
97. Harboe, M., et al., The role of properdin in zymosan- and Escherichia coli-induced complement activation. J Immunol, 2012. 189(5): p. 2606-13.

98. Zarantonello, A., et al., An Ultrahigh-Affinity Complement C4b-Specific Nanobody Inhibits In Vivo Assembly of the Classical Pathway Proconvertase. The Journal of Immunology, 2020: p. ji2000528.

99. Jensen, R.K., et al., A potent complement factor C3-specific nanobody inhibiting multiple functions in the alternative pathway of human and murine complement. J Biol Chem, 2018. 293(17): p. 6269-6281.

100. Pedersen, H., et al., A C3-specific nanobody that blocks all three activation pathways in the human and murine complement system. J Biol Chem, 2020. 295(26): p. 8746-8758.

101. Pedersen, H., et al., A Complement C3-Specific Nanobody for Modulation of the Alternative Cascade Identifies the C-Terminal Domain of C3b as Functional in C5 Convertase Activity. J Immunol, 2020.

102. Wen, Y., et al., Structural evaluation of a nanobody targeting complement receptor Vsig4 and its cross reactivity. Immunobiology, 2017. 222(6): p. 807-813.

103. Cruz, J.W., et al., A novel bispecific antibody platform to direct complement activity for efficient lysis of target cells. Sci Rep, 2019. 9(1): p. 12031.

104. Thielens, N.M., et al., C1q: A fresh look upon an old molecule. Molecular Immunology, 2017. 89: p. 73-83.

105. Pan, Q., R.O. Ebanks, and D.E. Isenman, Two Clusters of Acidic Amino Acids Near the NH<sub $>2</$ sub $>$ Terminus of Complement Component C4 $\alpha^{\prime}$-Chain Are Important for C2 Binding. The Journal of Immunology, 2000. 165(5): p. $2518-2527$.

106. Mortensen, S., J.K. Jensen, and G.R. Andersen, Solution Structures of Complement C2 and Its C4 Complexes Propose Pathwayspecific Mechanisms for Control and Activation of the Complement Proconvertases. The Journal of biological chemistry, 2016. 291(32): p. 16494-16507.

107. Xue, X., et al., Regulator-dependent mechanisms of C3b processing by factor I allow differentiation of immune responses. Nature Structural \& Molecular Biology, 2017. 24(8): p. 643-651.

108. Carroll, M.C. and D.E. Isenman, Regulation of humoral immunity by complement. Immunity, 2012. 37(2): p. $199-207$.

109. Pedersen, D.V., et al., Crystallization and X-ray analysis of monodisperse human properdin. Acta Crystallographica Section F, 2019. 75(2).

110. Pedersen, D.V., et al., Functional and structural insight into properdin control of complement alternative pathway amplification. EMBO J, 2017. 36(8): p. 1084-1099.

111. Laursen, N.S., et al., Substrate recognition by complement convertases revealed in the C5-cobra venom factor complex. EMBO J, 2011. 30(3): p. 606-16.

112. Schatz-Jakobsen, J.A., D.V. Pedersen, and G.R. Andersen, Structural insight into proteolytic activation and regulation of the complement system. Immunol Rev, 2016. 274(1): p. 59-73.

113. Pangburn, M.K., Analysis of the natural polymeric forms of human properdin and their functions in complement activation. The Journal of Immunology, 1989. 142(1): p. 202-207.

114. Wiesmann, C., et al., Structure of C3b in complex with CRIg gives insights into regulation of complement activation. Nature, 2006. 444(7116): p. 217-20.

115. Wang, X., et al., Prevention of Fatal C3 Glomerulopathy by Recombinant Complement Receptor of the Ig Superfamily. J Am Soc Nephrol, 2018. 29(8): p. 2053-2059.

116. Zheng, F., et al., Molecular imaging with macrophage CRIg-targeting nanobodies for early and preclinical diagnosis in a mouse model of rheumatoid arthritis. J Nucl Med, 2014. 55(5): p. 824-9.

117. Williams, R.O., Collagen-induced arthritis in mice. Methods Mol Med, 2007. 136: p. 191-9.

118. Monach, P.A., D. Mathis, and C. Benoist, The K/BxN arthritis model. Curr Protoc Immunol, 2008. Chapter 15: p. Unit 15.22.

119. Zheng, F., et al., Specificity Evaluation and Disease Monitoring in Arthritis Imaging with Complement Receptor of the Ig superfamily targeting Nanobodies. Sci Rep, 2016. 6: p. 35966. 
120. Bernth Jensen, J.M., et al., Abundant human anti-Gala3Gal antibodies display broad pathogen reactivity. Sci Rep, 2020. 10(1): p. 4611.

121. Bernth Jensen, J.M., et al., Complement activation by human IgG antibodies to galactose- $\alpha$-1,3-galactose. Immunology, 2020. $\mathbf{n} / \mathbf{a}(\mathrm{n} / \mathrm{a})$.

122. Lausen, M., et al., Analysis of complement deposition and processing on Chlamydia trachomatis. Med Microbiol Immunol, 2020.

123. Pedersen, D.V., et al., Recruitment of properdin by bi-specific nanobodies activates the alternative pathway of complement. Molecular Immunology, 2020. 124: p. 200-210.

124. Ricklin, D., et al., The renaissance of complement therapeutics. Nature Reviews Nephrology, 2017. 14: p. 26.

125. Steeland, S., R.E. Vandenbroucke, and C. Libert, Nanobodies as therapeutics: big opportunities for small antibodies. Drug Discovery Today, 2016. 21(7): p. 1076-1113.

126. Alawieh, A., E.F. Langley, and S. Tomlinson, Targeted complement inhibition salvages stressed neurons and inhibits neuroinflammation after stroke in mice. Sci Transl Med, 2018. 10(441).

127. Chatterjee, P., et al., Complement C4 maintains peripheral B-cell tolerance in a myeloid cell dependent manner. European journal of immunology, 2013. 43(9): p. 2441-2450. 